\title{
Cómo valorar la internacionalidad de las revistas de Ciencias Humanas y su categorización en ERIH
}

\author{
Adelaida Román-Román*, Elea Giménez-Toledo*
}

Resumen: La internacionalidad de las revistas científicas es un elemento de la calidad que cada vez es más considerado por las agencias de evaluación de los méritos científicos. Su análisis y evaluación plantea serias dificultades, especialmente en las Humanidades. Se propone por ello, tras un repaso de los indicadores más utilizados en algunos de los trabajos publicados entre 1997 y 2007, aplicar aquellos que resultaron de utilización más frecuente y fueron menos controvertidos, a un conjunto de revistas científicas españolas de Humanidades editadas por las universidades y por el CSIC.

Se analizan en las revistas seleccionadas la composición internacional de los consejos científicos, la internacionalidad de las contribuciones publicadas, y la presencia en bases de datos internacionales. Se aportan los resultados obtenidos por las revistas estudiadas y se inicia la discusión a partir de la comparación de éstos con los obtenidos por las mismas revistas en los listados ERIH, analizando las posibles causas de las discrepancias más destacables observadas. Se concluye con la necesidad de definir mejor y ampliar los indicadores para determinar de manera mas ajustada y objetiva los niveles de internacionalidad de las revistas, tanto en la elaboración de las listas ERIH, como, en general, en la valoración de los niveles de internacionalidad de las revistas de Humanidades, habida cuenta de la complejidad del fenómeno y de la conveniencia de que los procesos de evaluación estén basados en lo posible en mecanismos que puedan ser reproducibles.

Palabras clave: Revistas científicas, Humanidades, evaluación científica, indicadores de internacionalidad, ERIH.

\section{How to assess journals' internationality in the Humanities and their categorization in ERIH}

Abstract: The internationality of scholarly journals is an important aspect of scientific quality and is considered ever more highly by funders and assessment agencies. But the analysis of different levels of internationality of publications is a complex task,

* G. I. Evaluación de Publicaciones Científicas. Instituto de Estudios Documentales sobre Ciencia y Tecnología (IEDCYT). Centro de Ciencias Humanas y Sociales (CCHS). Consejo Superior de Investigaciones Científicas (CSIC). Correo-e: adelaidar@telefonica.net; elea.gimenez@cchs.csic.es.

Recibido: 23-7-2009; 2. ${ }^{a}$ versión: 15-12-2009; aceptado: 28-2-2010. 
particularly in the Humanities. This paper attempts to make a small contribution toward clarifying some of the methodological difficulties encountered.

After a brief review of indicators on journal internationality employed in a number of papers published between 1997 and 2007, the authors selected several that were among the most frequently used and least controversial. The indicators were then applied to Spanish scholarly journals to assess their internationality through the following parameters: internationality of advisory boards, internationality of contributing authors and international visibility. The results obtained in this exercise are presented and in the discussion they are compared with the categorization assigned to the same journals in the ERIH "Initial lists". Possible reasons for the major discrepancies observed are analyzed.

The conclusions point to the urgent need to clarify the applied indicators to determine internationality, to expand their number and to assure their objectivity, in order to guarantee the reproducibility of the assessment process and results.

Keywords: Scholarly journals, Humanities, scientific assessment, internationality indicators, ERIH.

\section{Introducción}

Desde mediados de la década de los noventa hasta nuestros días, el interés por los estudios orientados a analizar e incluso a medir la internacionalidad de las publicaciones científicas ha ido en aumento y en paralelo al proceso de internacionalización de la ciencia. Fruto de este interés ha sido la creciente publicación de estudios proponiendo diferentes metodologías, no ya para el análisis de la internacionalidad de las revistas y de los artículos en ellas publicados, sino para su posible evaluación. En el ámbito de las Humanidades y las Ciencias Sociales, en las que la internacionalidad de los objetos de estudio no es siempre evidente, la valoración del grado de internacionalidad de una revista no es asunto fácil. Dada la relevancia que se otorga hoy a la internacionalidad de las revistas, relacionándola con la calidad en los procesos de evaluación de la producción científica, parece interesante y oportuno asomarse a algunos de los trabajos publicados en la década 1997-2007 y repasar el tipo de aproximaciones metodológicas utilizadas para su valoración. Ello ayudará a optar por algunos de los indicadores utilizados para aplicarlos a una selección de revistas españolas de Humanidades de carácter académico, con el objetivo de contrastar su utilidad en la determinación de la internacionalidad de las revistas. Asimismo, parece conveniente ponerlos en relación con aquellos empleados por el European Reference Index for the Humanities (ERIH). Este proyecto es el primer intento europeo por categorizar las revistas de Humanidades en las que se publica la investigación europea. Está impulsado por la European Science Foundation (ESF) y en sus inicios también estuvo financiado por el proyecto ERA-NET de la Comisión Europea HERA (Humanities in the European Research Area). Las revistas son categorizadas en A, B o C, según su nivel de internacionalidad y a partir de la opinión de 15 paneles de expertos que reciben, además, los comentarios de centros de investigación, asociaciones temáticas y agencias de financiación. Por otra parte, los editores de 
revistas tienen la posibilidad de defender la inclusión de una revista en ERIH o de presentar argumentos para cambiar de categoría, lo que significa que la construcción de ERIH pretende dar vOz a los distintos implicados en el proceso.

\section{Antecedentes}

Una de las primeras aproximaciones en la década señalada se orienta a la valoración de la visibilidad internacional de las revistas nacionales como una parte del estudio de la internacionalidad (Pérez Álvarez-Ossorio y otros, 1997). Los autores definen la difusión internacional (ID) de la producción nacional en un campo dado como el porcentaje de los artículos de una disciplina y en un período concreto, recogidos por una base de datos nacional que son también recogidos por una base de datos internacional especializada.

Este aspecto de la visibilidad internacional ha sido trabajado por diversos autores con aproximaciones diferentes y, muy frecuentemente, analizando la presencia de las revistas en las bases de datos Web of Science (WoS) como paradigma de base de datos que recogen las revistas que constituyen la "corriente principal" de la ciencia. Un ejemplo es el trabajo sobre la visibilidad de las revistas chinas de ciencias (Ren y Rousseau, 2002). Los indicadores que aquí se emplean para medir la visibilidad internacional de las revistas son las pautas de citación y la composición de los consejos editoriales de las revistas incluidas en WoS, señalando la baja proporción de miembros extranjeros en ellas, así como el escaso número de contribuciones extranjeras en los artículos que publican. Esto, unido a los niveles bajos de citación, hace que, a pesar de estar incluidas en WoS, se aprecien unos niveles de internacionalidad muy escasos.

Un planteamiento similar se da en el interesante trabajo de Gutiérrez Puebla (1999), pues se manejan también dos de esas variables — publicación de artículos de alto nivel científico procedentes de todo el mundo y composición internacional del consejo editorial - aplicadas, además, a revistas ya consideradas "internacionales" por figurar con factores de impacto altos/medios en el Social Sciences Citation Index (SSCI). Se realiza el análisis sobre 19 revistas, todas ellas editadas en los Estados Unidos o en el Reino Unido y se excluyen aquéllas cuyo título haga suponer que tienen un carácter eminentemente nacional. Los resultados muestran que las contribuciones de los países no anglosajones solo representan un 12,1\% del total y que, en consecuencia, los niveles de internacionalidad en este sentido son muy reducidos, pues el Reino Unido y Estados Unidos aportan el grueso de la producción $(73,5 \%)$. En cuanto a la composición de los comités editoriales, resulta a grandes rasgos parecida a la derivada de las autorías: Estados Unidos, como promedio, representa un 40,99\% de los consejeros, y Reino Unido un 37,78\%, quedando poco margen para la participación de otros países. Se evidencia, por otra parte, la importancia que tiene la internacionalidad de los temas tratados por las revistas.

En la misma línea se encuentra el trabajo de Uzún (2004) para establecer los diferentes niveles de internacionalidad en cinco revistas de primer nivel especia-

Rev. Esp. Doc. Cient., 33, 3, julio-septiembre, 341-377, 2010. ISSN: 0210-0614. doi:10.3989/redc.2010.3.735 
lizadas en el campo de la Información Científica, la Bibliometría y la Cienciometría, editadas en distintos países y cubiertas por WoS. Los datos obtenidos permiten afirmar que el sector se encuentra en un proceso continuado y creciente de internacionalización. En esta conclusión es un factor importante el hecho de que el intercambio de contribuciones entre Europa y Estados Unidos es bidireccional, es decir, de importancia equivalente en los dos sentidos: hay contribuciones europeas en la revistas norteamericanas y de autores norteamericanos en las revistas europeas estudiadas. Es preciso señalar que la internacionalidad de una disciplina requiere de colaboraciones multidireccionales mutuas.

La internacionalidad de las contribuciones es también objeto de análisis en el interesante trabajo de Rey-Rocha y Martín-Sempere (2004). En él analizan seis revistas europeas de Ciencias de la Tierra, todas ellas cubiertas por una base de datos especializada de prestigio, GeoRef, y dos de ellas incluidas en Science Citation Index. No solo analizan las revistas con un alto porcentaje de artículos firmados por autores de instituciones extranjeras respecto de la institución editora y una alta proporción de contribuciones de autorías individuales extranjeras, sino que lo combinan con la baja proporción de artículos cuyo objeto de estudio es el territorio del propio país en el que se edita la revista.

En el campo de las Humanidades cabe destacar el trabajo de Malalana y otros (2007), que estudia la visibilidad internacional y la internacionalidad de las revistas españolas de Historia a través de los distintos indicadores que se han mencionado hasta ahora: presencia extranjera en los comités editoriales; presencia y distribución geográfica de instituciones extranjeras en las que trabajan los autores; autorías de trabajos individuales y coautorías; presencia de las revistas en catálogos y bases de datos internacionales; distribución temática y disciplinar de los artículos publicados por los autores extranjeros y la posible incidencia de la temática en el grado de "internacionalidad" de las revistas.

Los resultados permiten señalar la vinculación entre la especialidad temática y/o cronológica de la revista con los niveles de internacionalización de la misma, al menos en las de Historia. Además, se argumenta la repercusión que un consejo científico internacional puede tener tanto en la difusión como en la captación de originales, especialmente si sus miembros son agentes activos y no meros elementos de "prestigio". Finalmente, se incide en la importancia de las citas recibidas como indicador del uso y la influencia de la revista en la comunidad internacional de especialistas.

No se puede dejar de mencionar el estudio y la valoración crítica que se hace en el trabajo de Buela (Buela Casal y otros, 2006). Tras la aclaración de lo que significa el término "internacional" aplicado a una publicación científica, permite tener una recopilación bastante amplia de indicadores con comentarios que, al menos, hacen reflexionar sobre su mayor o menor adecuación a lo que se pretende medir. Finalmente, los autores hacen una selección de entre todos los indicadores descritos, para aplicarlos a cuatro revistas de Psicología con el objetivo de probar y contrastar la hipótesis de que son los indicadores mas idóneos: colaboración internacional; distribución multinacional de los miembros del comité 
editorial; distribución multinacional de los editores asociados; distribución multinacional de los autores. En sus conclusiones se reafirman en la distinción entre calidad e internacionalidad, como dos atributos diferentes, y mantienen un énfasis constante en ligar la internacionalidad a la presencia de diversos países, tanto en contribuciones como en consejos editoriales.

Como puede observarse, el núcleo de indicadores básicos que determinarían la internacionalidad de una revista está bastante delimitado, a la luz de los artículos citados y también de las orientaciones que se dan en guías y manuales dedicadas específicamente a valorar la calidad de las publicaciones desde una perspectiva integral (Román, 2001; Delgado y otros, 2006).

Por último, deben quedar expuestos aquí los criterios que la European Science Foundation ha establecido para poder construir el European Reference Index for the Humanities (ERIH) (ERIH Summary Guidelines, s.d.), primer listado europeo categorizado de revistas de Humanidades - aunque incluye alguna disciplina de las Ciencias Sociales-. ERIH establece tres categorías (A, B y C) que no implican mayor o menor calidad, sino diferentes niveles de internacionalidad. La inclusión en los listados, independientemente de su categoría, ya representaría el sello de calidad de la revista.

Más concretamente, en ERIH $^{1}$ se definen las siguientes características para considerar internacional una revista:

"International journals" (Categories A and B):

A journal is defined as international when the following requirements are fulfilled in addition to those that apply to all journals:

1) - A genuine, varied and regular international cohort of contributors and readership

- Consistently high-quality scholarly content

- Broad consensus within the field concerning international status and visibility

2) In addition, they will have some, though not necessarily all, of the following characteristics:

- Active international advisory board

- Openness to unsolicited contributions

- Highly discriminating and selective in the choice of articles published

- Published on time and to an agreed schedule

The difference between category $A$ and category B journals is likely to be the degree to which they conform to 1) above, and both the number of characteristics under 2) to which they conform as well as the degree of conformity. Generally, A journals should conform to more of these characteristics, and to a greater extent, than $B$ journals."

\footnotetext{
1 Ver: http://www.esf.org/index.php?eID=tx_nawsecuredl\&u=0\&file=fileadmin/be_user/research_areas/HUM/Documents/ERIH/Scope\%20Notes/ERIHRevisted_GUIDELINES.pdf\&t=124092109 3\&hash=3547770ce6a9f61d5debb8903f13ea72 [consultado el 27 de abril de 2009].
} 
Los criterios que ERIH establece para asignar la categoría "A" a una revista y, por tanto, para considerarla altamente internacional, coinciden parcialmente con los que han sido empleados en los estudios citados (contribuciones extranjeras, comité científico internacional, visibilidad internacional de la publicación, citas internacionales recibidas); sin embargo, hay que añadir la consideración de que tenga una buena reputación entre investigadores de distintos países. Esto introduce una variable, quizá subjetiva, pero al fin y al cabo procedente de expertos, que marcará los resultados alcanzados por las revistas en cuanto a sus niveles de internacionalidad.

Hasta ahora ERIH ha hecho públicos los denominados "listados iniciales" de revistas categorizadas. Próximamente se publicarán los listados definitivos, resultado de las propuestas iniciales de los grupos de expertos y de las reclamaciones y peticiones de revisión de categoría que han podido hacer los editores de revistas hasta la fecha. Una vez que esos listados definitivos estén publicados tendrán validez durante cuatro años y transcurrido este plazo podrán ser actualizados nuevamente, atendiendo a las consideraciones de los expertos y a las reclamaciones de los editores.

\section{Objetivos}

En este trabajo se pretenden aplicar a un conjunto reducido y escogido de revistas españolas de diferentes disciplinas de Humanidades, algunos indicadores utilizados frecuentemente en estudios previos para la valoración de la internacionalidad de las revistas, con el fin de comprobar su mayor o menor adecuación para ello, así como su expresividad. El ejercicio tiene un interés evidente, teniendo en cuenta que cada vez más, los sistemas de evaluación aplicados por las diversas agencias que gestionan y distribuyen fondos públicos a través de convocatorias para optar a la financiación de proyectos, y aquéllas encargadas de la acreditación del profesorado universitario y de la evaluación de los investigadores, incorporan la internacionalidad como un elemento relevante relacionado con la calidad científica. Es importante, por tanto, y cada vez más urgente, encontrar la manera más ajustada y más fiable de valorar los niveles de internacionalidad de las revistas de Humanidades, objetivo al que espera contribuir el presente trabajo.

\section{Metodología}

\subsection{Selección de las revistas}

Las revistas objeto de análisis pertenecen todas ellas al ámbito de las Humanidades, con la acepción admitida en la propia estructura disciplinar del CSIC: Arte, Arqueología y Prehistoria, Antropología, Filosofía, Filologías e Historia.

La selección se hizo en función de estos criterios: 1) que fueran editadas por 
alguna de las universidades españolas o por el CSIC; 2) que hubieran obtenido la categoría "A", bien en la lista de revistas categorizadas de la ANEP (Agencia Nacional de Evaluación y Prospectiva) ${ }^{2}$, bien en la categorización asignada por el Grupo de investigación de Evaluación de Publicaciones Científicas (EPUC) del $\mathrm{CSIC}^{3}$ a las revistas que son indizadas en la base de datos ISOC; 3) que fueran revistas con categoría "B" en las categorizaciones ANEP y EPUC. Se trataba de asegurar así que las revistas seleccionadas estuvieran consolidadas y fueran de calidad reconocida. Aplicando estos dos filtros, se obtuvo un conjunto de 36 revistas (tabla I).

TABLA I

Revistas españolas de Humanidades seleccionadas para el estudio

\begin{tabular}{|c|c|c|c|}
\hline Revistas seleccionadas & $\begin{array}{l}\text { Cat. } \\
\text { ISOC }\end{array}$ & $\begin{array}{l}\text { Cat. } \\
\text { ANEP }\end{array}$ & Números analizados \\
\hline Al-Qantara. Madrid & A & $A+$ & 2006, $27(1,2) ; 2005,26(2)$ \\
\hline Anuario de Estudios Americanos. Sevilla & A & A & $2006,63(1,2) ; 2006,62(2)$ \\
\hline Anuario de Estudios Medievales. Barcelona & A & A & $2006,36(1,2) ; 2005,35(2)$ \\
\hline Archivo Español de Arqueología. Madrid & A & $A+$ & 2006, 79 (193); 2005, $78(191,192)$ \\
\hline Archivo Español de Arte. Madrid & A & A & $2006,79(314-316)$ \\
\hline Asclepio. Madrid & A & A & $2006,58(1,2) ; 2005,57(2)$ \\
\hline $\begin{array}{l}\text { C.F.C. Estudios Griegos e Indoeuropeos. } \\
\text { Madrid }\end{array}$ & A & $\mathrm{B}$ & 2004 (14), 2005 (15), 2006 (16) \\
\hline C.F.C. Estudios Latinos. Madrid & A & $\mathrm{B}$ & $2005,25(2), 2006,26(1,2)$ \\
\hline Complutum. Madrid & A & A & 2004, (15), 2005 (16), 2006 (17) \\
\hline Cuadernos de Filología Italiana. Madrid & A & B & 2005 (12 y n. ${ }^{\circ}$ extra), 2006 (13) \\
\hline Cuadernos de Historia Moderna. Madrid & A & $\mathrm{B}$ & 2004 (29), 2005 (30), 2006 (31) \\
\hline Dynamis. Granada & A & $\mathrm{B}$ & $2004(24), 2005$ (25), 2006 (26) \\
\hline Emerita. Madrid & A & A & $2006,74(1,2) ; 2005,73(2)$ \\
\hline $\begin{array}{l}\text { Estudis. Revista de Historia Moderna. Va- } \\
\text { lencia }\end{array}$ & A & $\mathrm{C}$ & $2004(30), 2005$ (31), $2006(32)$ \\
\hline Faventia. Barcelona & A & $\mathrm{C}$ & $2005,27(2), 2006,28(1,2)$ \\
\hline Gerion. Madrid & A & C & $2005,23(2), 2006,24(1,2)$ \\
\hline Habis. Sevilla & A & $\mathrm{B}$ & 2004 (35), 2005 (36), 2006 (37) \\
\hline
\end{tabular}

2 Tal categorización fue llevada a cabo aplicando los criterios definidos en Criterios de calidad en la investigación en Humanidades: http://ciencia.micinn.fecyt.es/ciencia/anep/files/2007-criterioshh.pdf [consultado el 3 de septiembre de 2009].

3 Categorización para la admisión de revistas en la base de datos ISOC. Véase: http://bddoc. csic.es:8080/informacion.do?tabla=revi\&bd=ISOC\&estado_formulario=show\#info5 [consultado el 10 de octubre de 2009]. 
TABLA I (continuación)

\begin{tabular}{|c|c|c|c|}
\hline Revistas seleccionadas & $\begin{array}{l}\text { Cat. } \\
\text { ISOC }\end{array}$ & $\begin{array}{l}\text { Cat. } \\
\text { ANEP }\end{array}$ & Números analizados \\
\hline Hispania. Madrid & A & $\mathrm{A}+$ & $2006,66(222,223,224)$ \\
\hline Historia Agraria. Murcia; Barcelona & A & $\mathrm{A}+$ & $2006,16(38,39,40)$ \\
\hline $\begin{array}{l}\text { Minerva. Revista de Filología Clásica. Va- } \\
\text { lladolid }\end{array}$ & $\mathrm{A}$ & $\mathrm{C}$ & 2006 (19), 2005 (18), 2004 (17) \\
\hline Misc. Est. Ar. y Heb. Secc. Hebreo. Granada & A & $\mathrm{C}$ & 2006 (55), 2005 (54); $2004(53)$ \\
\hline Rev Dialectología y T. Populares. Madrid & $\mathrm{B}$ & A & $2006,61(1,2) ; 2005,61(2)$ \\
\hline $\begin{array}{l}\text { Rev. Complutense de Historia de América. } \\
\text { Madrid }\end{array}$ & A & $\mathrm{A}+$ & 2004 (30), 2005 (31), 2006 (32) \\
\hline $\begin{array}{l}\text { Rev. Española de Antropología America- } \\
\text { na. Madrid }\end{array}$ & $\mathrm{B}$ & $\mathrm{B}$ & $2006,36(1,2) ; 2007,37$ (1) \\
\hline Revista de Antropología Social. Madrid & $\mathrm{B}$ & $\mathrm{B}$ & 2004 (13), 2005 (14), 2006 (15) \\
\hline Revista de Filología Alemana. Madrid & A & $\mathrm{A}+$ & 2004 (12), 2005 (13), 2006 (14) \\
\hline Revista de Filología Española. Madrid & A & A & $2006,86(1,2) ; 2005,85(2)$ \\
\hline Revista de Filosofia. Madrid & $\mathrm{B}$ & A & 2006, $30(2) ; 2006,31(1,2)$ \\
\hline Revista de Historia Económica. Madrid & A & $\mathrm{A}+$ & $2006,24(1,2,3)$ \\
\hline Revista de Indias. Madrid & A & $A+$ & $2006,66(222,223$ y 224) \\
\hline Revista de Literatura. Madrid & A & $\mathrm{B}$ & 2006, 68 (136 y 135); 2005, 67 (134) \\
\hline Sefarad. Madrid & A & $\mathrm{A}+$ & 2006, $66(1,2) ; 2005,65(2)$ \\
\hline $\begin{array}{l}\text { Studia Historica. Historia Medieval. Sala- } \\
\text { manca }\end{array}$ & A & $\mathrm{C}$ & 2005 (23), 2006 (24) 2007 (25) \\
\hline $\begin{array}{l}\text { Studia Historica. Historia Moderna. Sala- } \\
\text { manca }\end{array}$ & A & C & 2005 (27), 2006 (28), 2007 (29) \\
\hline Trabajos de Prebistoria. Madrid & A & A & $2006,63(1,2) ; 2005,62(2)$ \\
\hline Zephyrus. Salamanca & A & $\mathrm{C}$ & 2005 (58), 2006 (59), 2007 (60) \\
\hline
\end{tabular}

\subsection{Los indicadores de internacionalidad}

De todos los indicadores que describe la bibliografía especializada, se han seleccionado algunos de los utilizados con más frecuencia que, por una parte, parecían de una objetividad incuestionable y, por otra, era viable aplicar en un marco de tiempo razonable. Las autoras proponen fórmulas propias basadas en esos indicadores dirigidas muy explícitamente a valorar la internacionalidad de tres aspectos básicos en una publicación científica: su política y su gestión editorial, analizada a través de la composición de sus Comités Científicos (CC); sus autores, cuya internacionalidad se establece a partir del análisis de sus instituciones de trabajo; y, finalmente, su visibilidad internacional, en función de su pre- 
sencia en las bases de datos bibliográficas internacionales más prestigiosas, tanto multidisciplinares como especializadas, y en su presencia en Internet. Esta última no podría ser considerada en sí misma un indicador de calidad, pero sí está relacionada con la posibilidad de ser vista, leída y citada; desde este punto de vista, una revista bien difundida en bases de datos internacionales que, además, tenga una buena visibilidad en Internet, tendrá sin duda una proyección hacia el exterior mucho mayor.

\subsubsection{Indicador de Internacionalidad de los Comités Científicos (IICC)}

La internacionalidad de los Comités Científicos o Asesores se mide en función de la composición de éstos, es decir, analizando la afiliación institucional (institución u organismo de pertenencia) de los miembros que lo forman. Un Comité Científico será tanto más internacional cuanto mayor sea la proporción de miembros de instituciones extranjeras que lo componen y cuanto mayor sea el número de países diferentes que participan en él. Las funciones que debe realizar el Comité Científico en términos de difusión de la revista en los distintos países representados en el Consejo y de atracción de autores de esos mismos países que contribuyan con sus artículos, pueden ser decisivos en relación con los niveles de internacionalidad que las revistas puedan alcanzar.

El indicador de internacionalidad de los Comités Científicos se formula de la siguiente manera:

$$
I I C C=y * x
$$

Donde $y$ representa al porcentaje de miembros de instituciones extranjeras en el Comité Científico y $x$ representa el número de países diferentes presentes en el Comité Científico exceptuando a España.

\subsubsection{Indicador de internacionalidad de las contribuciones publicadas (IICP)}

Este indicador pretende establecer los niveles de internacionalidad de los artículos publicados en una revista durante un período de tiempo o un conjunto de números editados. Se ha calculado teniendo en cuenta el conjunto de artículos publicados en los tres últimos números publicados de cada revista y el porcentaje de ellos que tienen al menos un autor firmante con una institución de trabajo de un país diferente al país editor. Por otra parte, se analizan los diferentes países que contribuyen con artículos en esos números. Finalmente, se ponen en relación el porcentaje de artículos con al menos un autor firmante de una institución extranjera y el número de países diferentes que contribuyeron en la revista.

El cálculo del indicador se hace mediante la siguiente fórmula:

$$
I I C P=y * x
$$

Rev. Esp. Doc. Cient., 33, 3, julio-septiembre, 341-377, 2010. ISSN: 0210-0614. doi:10.3989/redc.2010.3.735 
Donde $y$ representa el porcentaje de artículos internacionales (firmados al menos por un autor de una institución extranjera) y $x$ representa el número de países diferentes, exceptuando a España, que contribuyeron con sus artículos.

\subsubsection{Visibilidad internacional}

En este trabajo se trata de valorar la difusión internacional de las revistas mediante el análisis de su presencia en las principales bases de datos bibliográficas internacionales, tanto multidisciplinares como especializadas. A diferencia de los parámetros anteriores, no se propone en este caso un indicador único de carácter numérico que sintetice la información relativa a la difusión en bases de datos. Lo que se ofrece es el número de bases de datos en que está incluida cada revista, así como la calidad de éstas, en términos de mayor o menor exigencia de calidad a las revistas para ser aceptadas por los productores de las bases de datos.

Se sigue para ello la categorización de las bases de datos realizada por el Grupo de Investigación de Evaluación de Publicaciones Científicas (Alcain y otros, 2008) al que pertenecen las autoras de este trabajo. Los criterios de categorización son los siguientes ${ }^{4}$ :

- Son bases de datos de categoría A las que exigen como mínimo que la revista tenga sistema de revisión por expertos, cumpla la periodicidad, e incluya tanto el lugar de trabajo de los autores como el resumen.

- Son bases de datos de categoría B aquéllas que exigen a las revistas que cumplan algunos criterios de los anteriores, pero no todos.

- Son bases de datos de categoría C las que sólo exigen que las revistas sean del área temática de la base de datos.

- Las bases que no aportan datos sobre los criterios aplicados para aceptar una revista, se consignan como SD (sin datos).

Una vez examinada la presencia de las revistas en bases de datos de una u otra categoría, se establecen cuatro tramos que atienden a su visibilidad: muy alta (presentes en 3 o 4 bases de datos de categoría A y en algunas del resto de categorías), alta (presentes en 2 bases de datos de categoría A y en una o varias del resto de categorías), media (presentes en 1 base de datos de categoría A y en una o varias del resto de categorías) o baja (no presentes en bases de datos de categoría A, pero presentes en una o más bases de datos del resto de categorías).

${ }^{4}$ Cada una de estas categorías recibe una puntuación diferente en el sistema de valoración integrada de revistas de Ciencias Sociales y Humanas que ofrece la plataforma RESH (http://resh. cindoc.cisc.es). En esa valoración integrada se combinan distintos indicadores de calidad y da lugar a un ranking de revistas; por ello, cada indicador debe tomar un valor numérico (Alcain y otros, 2008). En este artículo se ha decidido prescindir de esas puntuaciones, empleando únicamente el número de bases de datos de cada categoría en que están recogidas las publicaciones. 
El establecimiento de tramos —al igual que el de pesos específicos para cada variable- podría considerarse arbitrario, pero en el caso de la presencia en bases de datos internacionales los tramos establecidos marcan bien las diferencias en la visibilidad, y es esto precisamente lo que se pretende.

Además, como se indicaba anteriormente, se considera también el hecho de que la revista ofrezca el texto completo de sus artículos en Internet, así como un archivo histórico de artículos de al menos cinco años. No se asigna una baremación a esta variable, sino que simplemente se ofrece como un dato más para matizar la visibilidad de las revistas.

\subsubsection{Categorización final de internacionalidad a partir de las tres variables analizadas}

Una vez obtenidos los valores para cada una de las variables analizadas, se distribuyen en tres tramos cada una de ellas, empleando para ello percentiles (percentil 1: 0,33; percentil 2: 0,66, excepto para Visibilidad internacional en que se toma el valor 0,60 para posibilitar el establecimiento de tres tramos; percentil 3: 1. Véase tabla VI). De esta manera, se puede reducir el conjunto de valores posibles a tres: 1, 2 y 3, que se corresponderían con niveles bajo, medio y alto respectivamente de cada variable en función de la muestra analizada. La suma de los nuevos valores (percentiles) asignados a cada una de las tres variables dará lugar a una nueva columna (véase tabla V) sobre cuya distribución se volverán a calcular y a aplicar percentiles siguiendo el criterio anteriormente comentado. Los resultados se equipararán del siguiente modo: el 3 con la categoría A (internacionalidad alta), el 2 con la categoría B (internacionalidad media) y el 1 con la categoría C (internacionalidad baja). Una vez reducidas las variables a esta categorización (denominada "internacionalidad EPUC») será posible establecer la comparación con la categorización ERIH.

\section{Resultados}

\subsection{Internacionalidad de los Comités Científicos}

Como se mencionaba anteriormente, la internacionalidad de los Comités Científicos se ha estudiado desde dos dimensiones: el número y porcentaje de los miembros pertenecientes a instituciones extranjeras que forman parte de los Consejos y el número de países diferentes que están representados en ellos. Así, la internacionalidad de un consejo científico vendría dada por el producto de ambos parámetros.

La tabla II muestra los resultados obtenidos para este indicador. Se incorpora una columna IICC/100 que permite trabajar con una cifras más manejables.

La distribución de las revistas atendiendo al porcentaje de miembros extranjeros en sus Comités Científicos quedaría como se muestra en la figura 1. 


\begin{tabular}{|c|c|c|c|c|c|c|c|c|c|c|c|c|c|c|}
\hline & U⿺辶日: & $\underset{\substack{n \\
c}}{\infty}$ & 8) & $\begin{array}{l}\infty \\
\infty \\
i\end{array}$ & $\stackrel{10}{-}$ & $\begin{array}{l}8 \\
\text { n. }\end{array}$ & 운 & $\begin{array}{l}\hat{\sigma} \\
i n\end{array}$ & 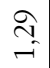 & $\begin{array}{l}8 \\
\forall\end{array}$ & $\stackrel{8}{-}$ & $\stackrel{\approx}{\sim}$ & 8 & $\begin{array}{l}n \\
\infty \\
-i\end{array}$ \\
\hline & U્ & $\begin{array}{l}\infty \\
\stackrel{\infty}{=} \\
\stackrel{0}{\sim}\end{array}$ & $\begin{array}{l}8 \\
80 \\
0\end{array}$ & $\begin{array}{l}8 \\
\infty \\
\infty \\
\text { N }\end{array}$ & $\stackrel{\overrightarrow{1}}{\stackrel{+}{6}}$ & $\begin{array}{l}8 \\
8 \\
8 \\
8\end{array}$ & $\stackrel{n}{\stackrel{n}{a}}$ & $\begin{array}{l}\sqrt{6} \\
\stackrel{0}{0} \\
i n\end{array}$ & 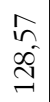 & $\begin{array}{l}8 \\
8 \\
8 \\
8\end{array}$ & $\begin{array}{l}8 \\
8 \\
0 \\
-1\end{array}$ & 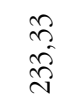 & $\begin{array}{l}8 \\
8 \\
8\end{array}$ & $\begin{array}{c}\mathcal{O} \\
+ \\
\infty \\
-\end{array}$ \\
\hline 0 & 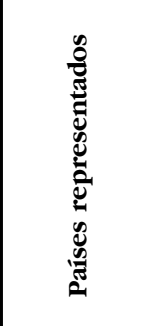 & 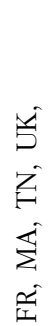 & 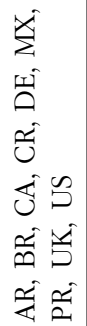 & 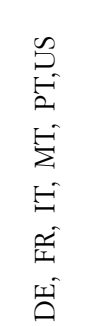 & 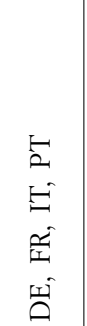 & 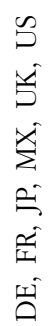 & 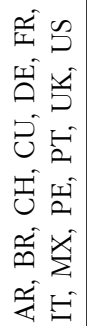 & 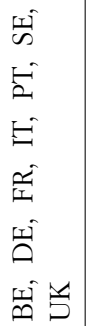 & 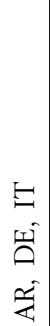 & 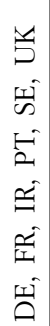 & 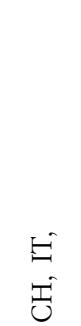 & 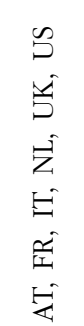 & 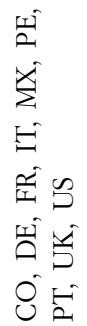 & 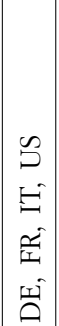 \\
\hline 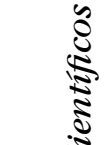 & 乙造总 & $\psi$ & $a$ & 6 & 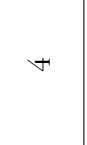 & 6 & $\stackrel{\mathcal{I}}{\sim}$ & $r$ & $n$ & 0 & $\sim$ & 6 & $a$ & $\uplus$ \\
\hline$=\frac{\mathscr{3}}{3}$ & ○ & $\begin{array}{l}\text { in } \\
\text { in }\end{array}$ & $\begin{array}{r}\approx \\
n\end{array}$ & 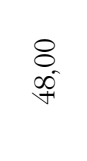 & $\stackrel{\infty}{\underset{F}{\rightleftarrows}}$ & $\begin{array}{l}8 \\
8 \\
0\end{array}$ & $\begin{array}{l}\text { a) } \\
\text { î }\end{array}$ & $\begin{array}{l}\stackrel{n}{\hat{\sigma}} \\
\infty\end{array}$ & $\begin{array}{l}8 \\
\infty \\
\text { if }\end{array}$ & $\begin{array}{l}\hat{6} \\
8 \\
0\end{array}$ & $\begin{array}{l}8 \\
\infty \\
\infty\end{array}$ & $\begin{array}{l}\infty \\
\infty \\
\infty \\
\end{array}$ & $\begin{array}{l}\hat{\sigma} \\
8 \\
\delta\end{array}$ & 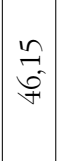 \\
\hline 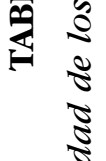 & 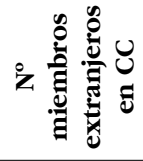 & 6 & $\widetilde{(}$ & $\underset{\sim}{\simeq}$ & $r$ & $\infty$ & $\approx$ & $\nexists$ & $n$ & $\infty$ & $\psi$ & $r$ & $\stackrel{ }{\circ}$ & 6 \\
\hline 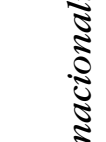 & 记 & $\exists$ & ì & $\stackrel{n}{\sim}$ & 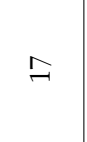 & $\stackrel{0}{\sim}$ & $\hat{n}$ & $\vec{\sim}$ & 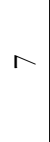 & $\mathcal{I}$ & in & $\stackrel{\infty}{\sim}$ & $\stackrel{\text { In }}{\sim}$ & $\stackrel{n}{\pi}$ \\
\hline 5 & 萢莺 & $\stackrel{U}{\tilde{U}}$ & $\frac{U}{\tilde{U}}$ & $\stackrel{U}{\tilde{U}}$ & $\stackrel{\cup}{\mathcal{O}}$ & 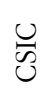 & $\stackrel{\cup}{\mathscr{U}}$ &  & $\sum_{S}$ & $\sum_{0}$ & 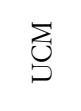 & 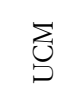 & 象 & $\begin{array}{l}0 \\
\tilde{U}\end{array}$ \\
\hline & 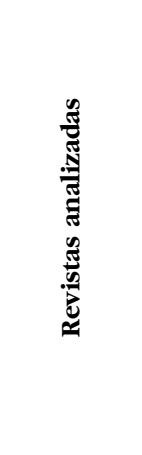 & 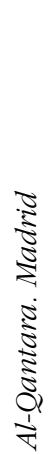 & 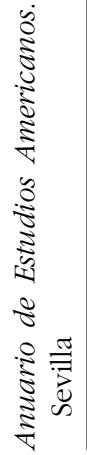 & 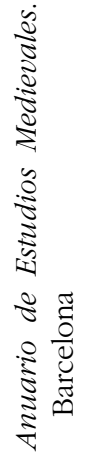 & 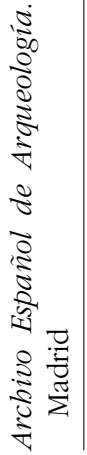 & 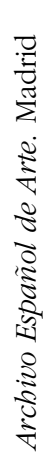 & 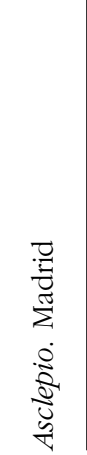 & 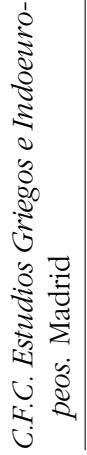 &  & 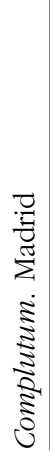 & 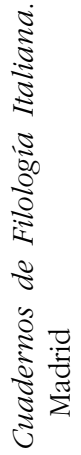 & 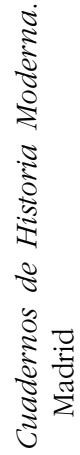 & 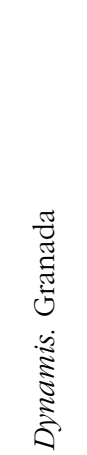 & 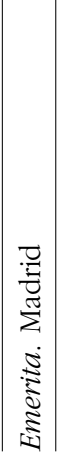 \\
\hline
\end{tabular}




\begin{tabular}{|c|c|c|c|c|c|c|c|c|c|c|c|c|c|c|}
\hline 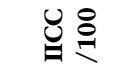 & $\stackrel{\curvearrowright}{r}$ & $\begin{array}{l}\stackrel{2}{n} \\
\text { ni }\end{array}$ & $\begin{array}{l}8 \\
\text { in }\end{array}$ & 8 & $\stackrel{\infty}{\sim}$ & $\begin{array}{l}\text { oे } \\
\text { å }\end{array}$ & $\begin{array}{l}8 \\
0\end{array}$ & $\begin{array}{l}8 \\
0\end{array}$ & $\begin{array}{l}\hat{i} \\
\text { ì }\end{array}$ & $\begin{array}{l}8 \\
\text { ri }\end{array}$ & $\underset{\sigma}{\tilde{\sigma}}$ & $\vec{i}$ & $\underset{+i}{8}$ & $\begin{array}{l}8 \\
\text { in }\end{array}$ \\
\hline U্ট & $\begin{array}{l}n \\
i n \\
\infty \\
N\end{array}$ & $\begin{array}{l}8 \\
0 \\
n \\
n\end{array}$ & $\begin{array}{l}8 \\
8 \\
8\end{array}$ & & $\begin{array}{l}\text { ơ } \\
\infty \\
\\
\sim\end{array}$ & $\begin{array}{l}R \\
\stackrel{2}{0} \\
\stackrel{2}{\alpha}\end{array}$ & $\begin{array}{l}8 \\
0\end{array}$ & $\begin{array}{l}8 \\
0\end{array}$ & $\begin{array}{l}\hat{n} \\
\infty \\
\tilde{u}^{0}\end{array}$ & $\begin{array}{l}8 \\
8 \\
8\end{array}$ & $\begin{array}{l}\text { त̃ } \\
\text { तु } \\
\text { J }\end{array}$ & $\begin{array}{l}\infty \\
\infty \\
\stackrel{0}{N}\end{array}$ & $\begin{array}{l}8 \\
8 \\
8\end{array}$ & $\begin{array}{l}8 \\
8 \\
8 \\
i n\end{array}$ \\
\hline 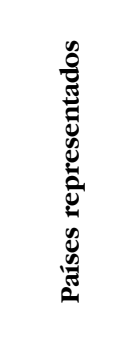 & 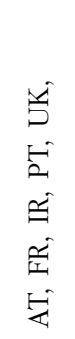 & 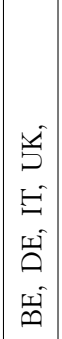 & 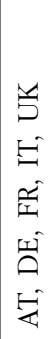 & & 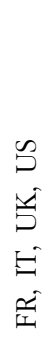 & 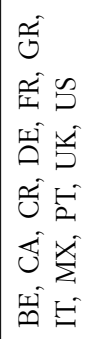 & & & 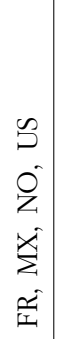 & 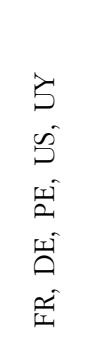 & 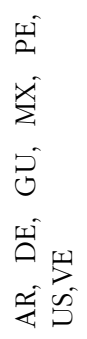 & 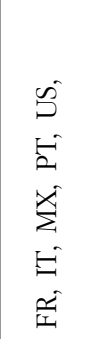 & 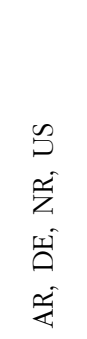 & 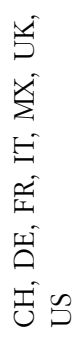 \\
\hline 乙象总 & in & 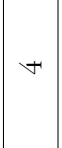 & in & 岕 & 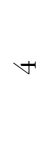 & $\exists$ & 0 & 0 & $*$ & in & $\Lambda$ & in & $\psi$ & $r$ \\
\hline 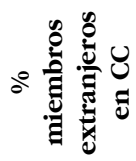 & 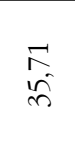 & $\begin{array}{l}0 \\
i n \\
\infty \\
\infty\end{array}$ & $\begin{array}{l}8 \\
8 \\
8\end{array}$ & & $\begin{array}{l}\text { So } \\
\text { in }\end{array}$ & $\begin{array}{l}\text { जi } \\
\text { i } \\
\infty\end{array}$ & 8 & 8 & $\begin{array}{l} \pm \\
\stackrel{-}{n}\end{array}$ & $\begin{array}{l}8 \\
8 \\
8\end{array}$ & $\begin{array}{l}\infty \\
\infty \\
\infty \\
\infty\end{array}$ & $\stackrel{F}{\stackrel{F}{n+1}}$ & $\begin{array}{l}8 \\
8 \\
8\end{array}$ & $\begin{array}{l}8 \\
8 \\
\infty\end{array}$ \\
\hline 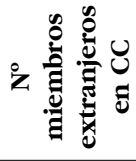 & $\stackrel{\circ}{\sim}$ & $r$ & $\infty$ & 岕 & $a$ & $\curvearrowright$ & 0 & 0 & $\infty$ & 6 & $\infty$ & $\ddot{\sim}$ & 6 & $\infty$ \\
\hline 乙产 U & $\stackrel{\infty}{\sim}$ & $\infty$ & $\infty$ & 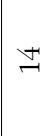 & $\stackrel{\sim}{\sim}$ & $\widetilde{\sim}$ & $\exists$ & $\stackrel{ }{\sim}$ & 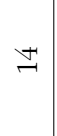 & $\stackrel{ }{\sim}$ & $a$ & $\stackrel{\vec{v}}{\sim}$ & 6 & $\stackrel{\circ}{-}$ \\
\hline 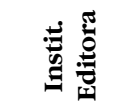 & 3 & 壱 & $\sum_{0}$ & $\mathscr{b}$ & 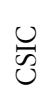 & $\sum_{\vdots}^{2}$ & $\overleftrightarrow{b}$ & 음 & $\stackrel{\cup}{\tilde{U}}$ & $\sum_{D}$ & $\sum_{S}$ & $\sum_{S}$ & $\sum_{S}$ & $\frac{U}{\vec{U}}$ \\
\hline 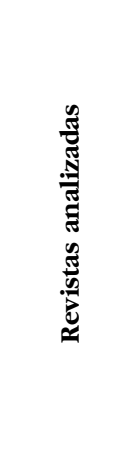 & 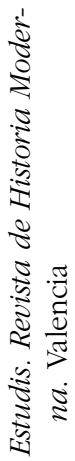 & 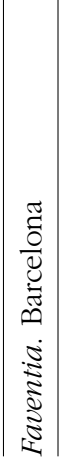 & 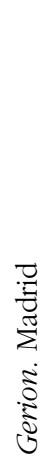 & 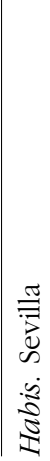 & 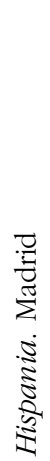 & 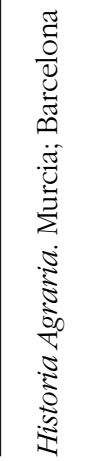 & 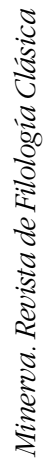 & 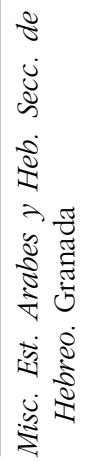 & 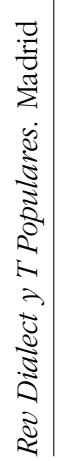 & 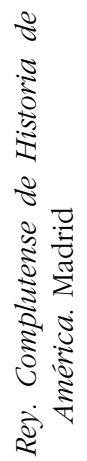 & 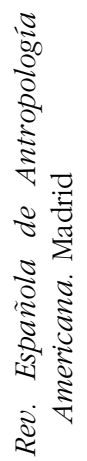 & 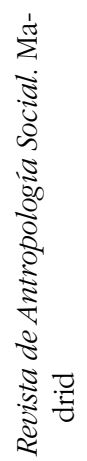 & 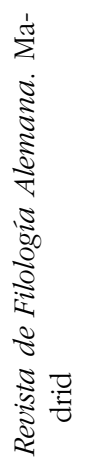 & 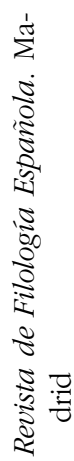 \\
\hline
\end{tabular}




\begin{tabular}{|c|c|c|c|c|c|c|c|c|c|}
\hline U⿺辶日: & $\underset{\text { ָิ }}{+}$ & oे & \& & $\begin{array}{l}\infty \\
\infty \\
i\end{array}$ & $\stackrel{8}{i n}$ & $\begin{array}{l}8 \\
\text { n }\end{array}$ & $\begin{array}{l}8 \\
0\end{array}$ & $\stackrel{\substack{n \\
\forall}}{f^{2}}$ & $\stackrel{m}{i}$ \\
\hline U্ট & $\begin{array}{l}8 \\
8 \\
\stackrel{1}{+}\end{array}$ & $\begin{array}{l}8 \\
\text { oे } \\
\text { in }\end{array}$ & $\begin{array}{l}8 \\
8 \\
0\end{array}$ & $\begin{array}{l}8 \\
8 \\
\infty \\
\text { i }\end{array}$ & $\begin{array}{l}8 \\
8 \\
17\end{array}$ & $\begin{array}{l}8 \\
8 \\
8\end{array}$ & & $\begin{array}{l}8 \\
10 \\
\stackrel{10}{+}\end{array}$ & $\frac{m}{N}$ \\
\hline 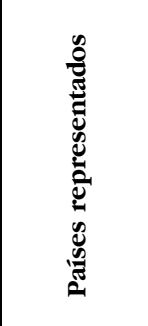 & 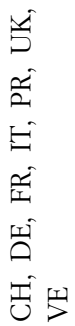 & 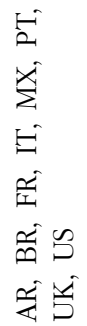 & 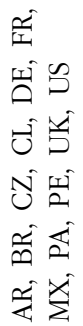 & 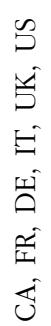 & 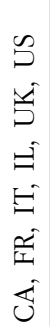 & 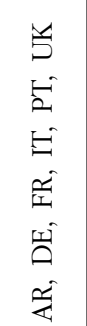 & & 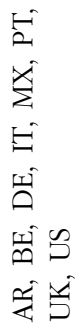 & 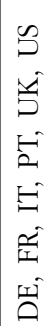 \\
\hline 乙 & $r$ & $\infty$ & $\exists$ & 0 & 0 & 6 & 岕 & $\infty$ & 6 \\
\hline o & $\begin{array}{l}8 \\
8 \\
8\end{array}$ & $\begin{array}{l}\mathbb{\sigma} \\
\approx \\
\sigma\end{array}$ & $\begin{array}{l}8 \\
8 \\
8\end{array}$ & $\begin{array}{l}\mathbf{6} \\
\dot{6} \\
+\end{array}$ & $\begin{array}{l}8 \\
\text { in }\end{array}$ & $\begin{array}{l}8 \\
\therefore \\
i n\end{array}$ & & $\begin{array}{l}\stackrel{0}{2} \\
\text { in }\end{array}$ & $\begin{array}{l}\text { if } \\
\text { in }\end{array}$ \\
\hline  & $\approx$ & $\underset{\beth}{\beth}$ & $\stackrel{\infty}{\sim}$ & $r$ & $a$ & $\infty$ & $\frac{\omega}{\omega}$ & $\curvearrowright$ & $\stackrel{ }{\circ}$ \\
\hline 记 & ㄱ. & $\widetilde{N}$ & in & $\stackrel{10}{-}$ & $\stackrel{\mathfrak{I}}{\exists}$ & $\stackrel{0}{\sim}$ & $\stackrel{\varpi}{\sim}$ & $\tilde{n}$ & $\tilde{N}$ \\
\hline 蔤 & $\sum_{0}$ & 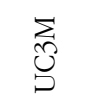 & $\stackrel{u}{\widetilde{v}}$ & $\stackrel{u}{\tilde{S}}$ & $\frac{u}{3}$ & 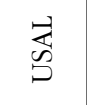 & 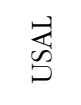 & $\stackrel{\cup}{\mathscr{O}}$ & 岕 \\
\hline 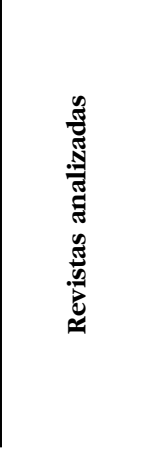 & 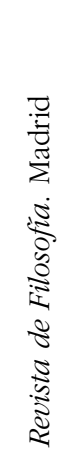 & 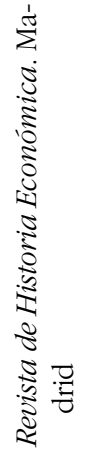 & 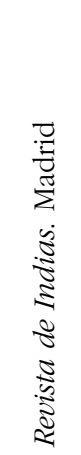 & 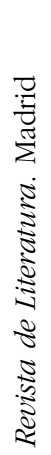 & 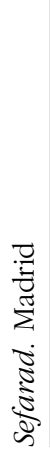 & 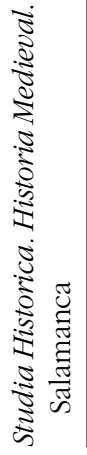 & 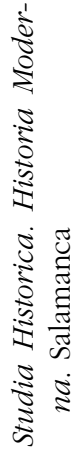 & 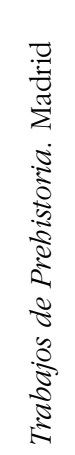 & 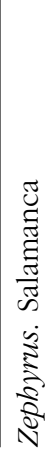 \\
\hline
\end{tabular}




\section{FIGURA 1}

Distribución de las revistas según el porcentaje de miembros extranjeros en sus Comités Científicos

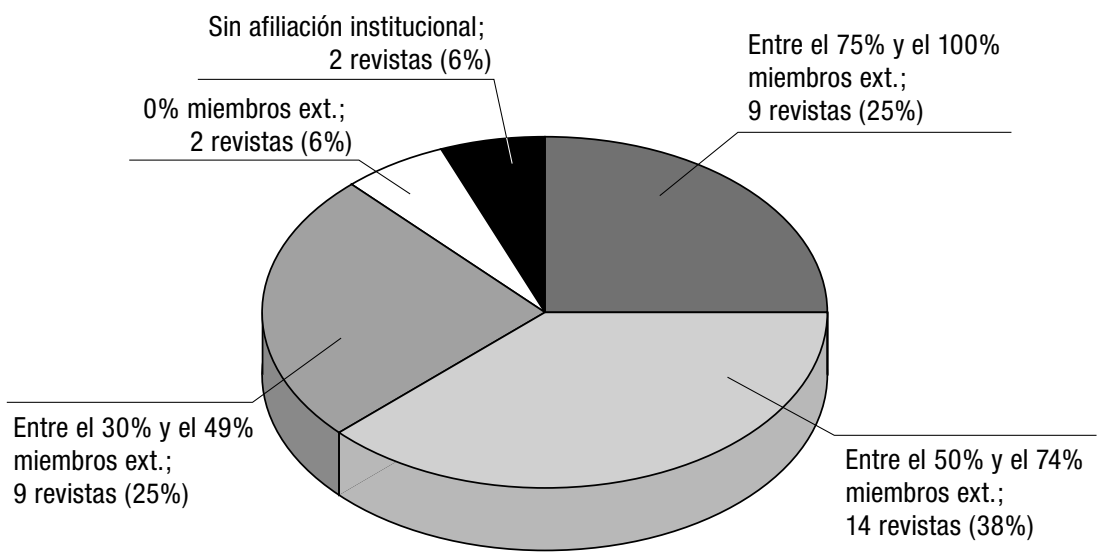

FIGURA 2

Distribución de las revistas según el número de países diferentes representados en sus Comités Científicos

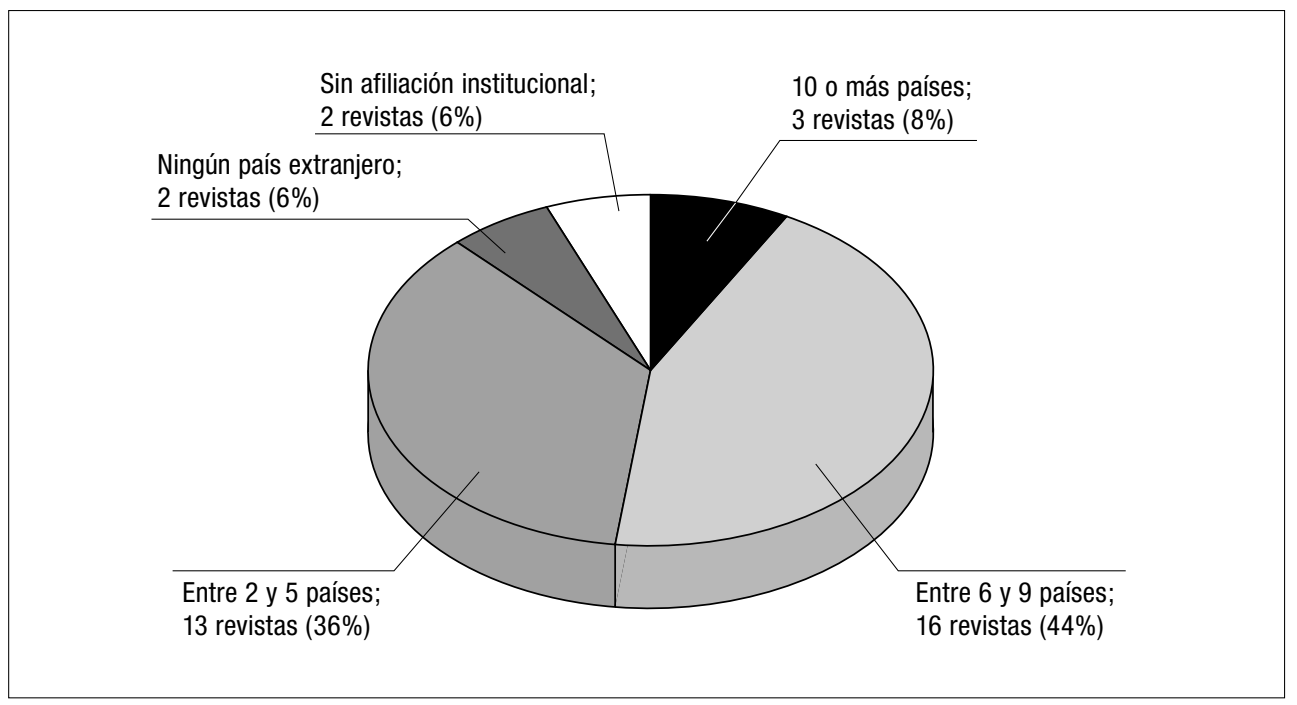


El conjunto de revistas analizadas cuenta con un total de 611 miembros en sus Comités Científicos, de los cuales un 54\% son extranjeros. Como promedio las revistas analizadas cuentan con un 59\% de miembros extranjeros.

Si se analizan ahora los resultados desde la segunda variable - la presencia de un mayor o menor número de países en los Comités Científicos- los resultados se muestran en la figura 2.

\subsection{Internacionalidad de las contribuciones publicadas}

La capacidad de atracción que tienen las revistas para captar trabajos de especialistas de los diferentes países depende de muchos factores: su prestigio entre le comunidad científica internacional del área, la extensión de ésta en número de especialistas, la situación que la revista ocupa en los índices de impacto y en las diversas categorizaciones elaboradas por organismos científicos prestigiosos, la naturaleza de la disciplina o sub-disciplina abordada por la revista (su carácter más internacional o más local, en términos generales), la actividad desarrollada por los miembros internacionales de su comité científico para conseguir contribuciones de los mejores especialistas del país al que él mismo pertenece, etc.

Desde este punto de vista, una revista será más internacional cuanta más capacidad de atracción tenga de firmas cualificadas de los más variados países. Por ello, una vez más, la internacionalidad de una revista en relación con los artículos que publica tendrá también dos variables: una será el número de artículos extranjeros publicados en un período dado y la otra será el número de países diferentes que aportaron sus trabajos a la revista en ese mismo período.

En este trabajo se ha considerado que un artículo es "internacional" si lo firma al menos un autor de una institución extranjera en relación al país editor de la revista.

En la tabla III pueden verse los resultados relativos a la internacionalidad de las contribuciones. Si se consideran los porcentajes de trabajos firmados al menos por un autor de una institución extranjera, las revistas se agrupan como muestra la figura 3.

Si se analizan los resultados desde el punto de vista del número de países extranjeros diferentes que contribuyeron publicando en las revistas, las revistas se distribuyen según la figura 4.

\subsection{Visibilidad internacional}

La visibilidad internacional de una revista incide de manera importante en las posibilidades que ésta tiene de ser utilizada, de ser leída. Las condiciones para que una revista pueda ser citada pasan, por ejemplo, por que $a$ ) sea conocida y b) sea accesible. Los títulos difundidos por las principales bases de datos inter- 


\begin{tabular}{|c|c|c|c|c|c|c|c|c|c|c|c|c|}
\hline \multirow{9}{*}{ 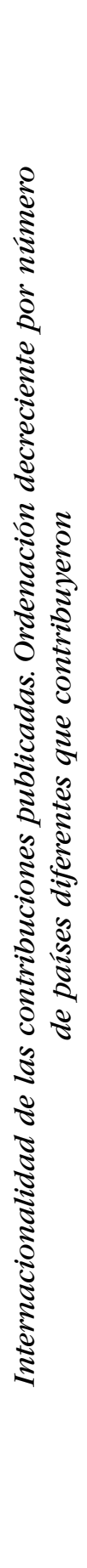 } & 气ે & $\begin{array}{l}\sigma^{\circ} \\
\infty\end{array}$ & $\begin{array}{l}n \\
\hat{6}\end{array}$ & $\stackrel{2}{\stackrel{\sigma}{*}}$ & $\underset{\sim}{n}$ & $\stackrel{n}{\sim}$ & $\begin{array}{l}\text { : } \\
i\end{array}$ & $\stackrel{\circ}{\rightarrow}$ & $\stackrel{\infty}{0}$ & $\begin{array}{l}\infty \\
\infty \\
\dot{n}\end{array}$ & \begin{tabular}{l}
$\infty$ \\
\multirow{0}{0}{}
\end{tabular} & $\begin{array}{l}8 \\
i\end{array}$ \\
\hline & 입 & $\begin{array}{l}\frac{+}{n} \\
\overrightarrow{8}\end{array}$ & \begin{tabular}{l}
$\infty$ \\
$\stackrel{2}{n}$ \\
\multirow{\sigma}{\sigma}{}
\end{tabular} & 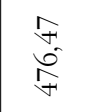 & $\begin{array}{l}\tilde{n} \\
\tilde{n} \\
\tilde{n}\end{array}$ & $\begin{array}{l}\tilde{n} \\
\stackrel{n}{n}\end{array}$ & $\begin{array}{l}\tilde{n} \\
\tilde{n} \\
\text { ปे }\end{array}$ & $\begin{array}{l}8 \\
8 \\
+\end{array}$ & $\begin{array}{l}\infty \\
\stackrel{1}{a} \\
\stackrel{1}{a}\end{array}$ & $\begin{array}{l}\stackrel{0}{1} \\
\stackrel{1}{\infty} \\
\infty\end{array}$ & $\frac{\infty}{N}$ & $\begin{array}{l}8 \\
8 \\
8\end{array}$ \\
\hline & 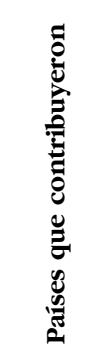 &  & 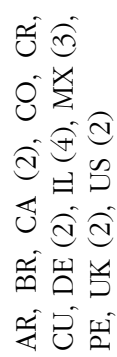 & 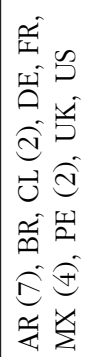 & 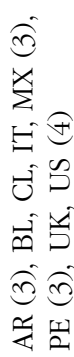 & 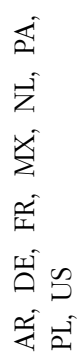 & 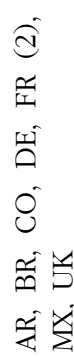 & 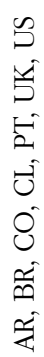 & 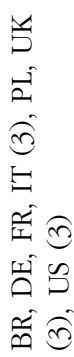 & 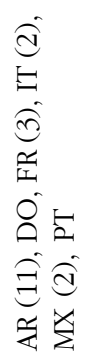 & 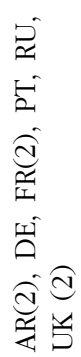 & 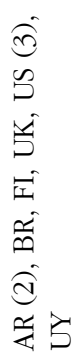 \\
\hline & 艺递总 & $\stackrel{\mathcal{Z}}{\sim}$ & $\stackrel{I}{\mathcal{V}}$ & $a$ & $\infty$ & $\infty$ & $r$ & $n$ & $\Lambda$ & 0 & 0 & 6 \\
\hline & 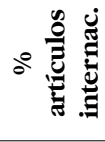 & $\stackrel{\Re}{i}$ & $\stackrel{\substack{n \\
i n}}{i=1}$ & ते & $\begin{array}{l}\hat{6} \\
\mathbb{b}_{0}\end{array}$ & $\begin{array}{l}\hat{\sigma} \\
\overrightarrow{\mathrm{i}}\end{array}$ & $\frac{n}{n}$ & $\begin{array}{l}8 \\
\text { : }\end{array}$ & $\stackrel{\infty}{\approx} \underset{\sim}{\sim}$ & $\begin{array}{l}\tilde{n} \\
\text { fí }\end{array}$ & ๙ & $\begin{array}{l}8 \\
\text { in }\end{array}$ \\
\hline & 艺总总 & $\stackrel{\infty}{\sim}$ & 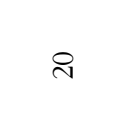 & $\stackrel{\infty}{\sim}$ & $\stackrel{\infty}{\sim}$ & $\infty$ & $\exists$ & $a$ & $\stackrel{n}{\sim}$ & 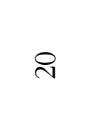 & $r$ & $\infty$ \\
\hline & 乙 & $\hat{\curvearrowright}$ & $\hat{n}$ & $\stackrel{m}{n}$ & $\widehat{\searrow}$ & in & ৯े & if & 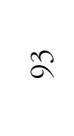 & $\vec{n}$ & in & $\stackrel{\bullet}{\sim}$ \\
\hline & 萢莺 & $\sum_{D}$ & كَّ & 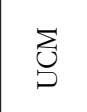 & $\stackrel{\cup}{\mathscr{U}}$ & 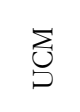 & $\stackrel{U}{\mathscr{U}}$ & $\sum_{S}$ &  & $\frac{U}{\tilde{U}}$ & $\begin{array}{l}\text { 岕 } \\
\text { 吕 }\end{array}$ & 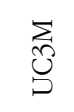 \\
\hline & 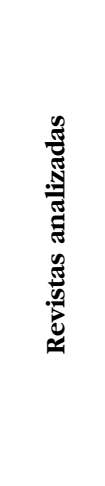 & 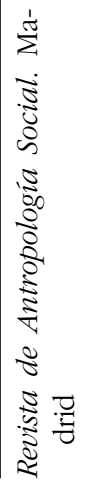 & 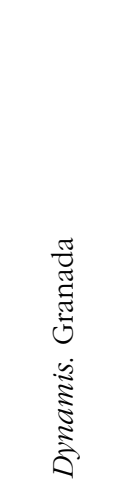 & 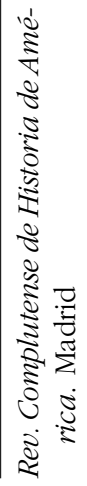 & 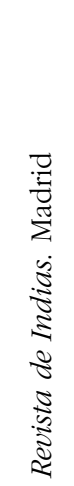 & 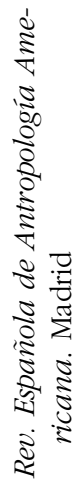 & 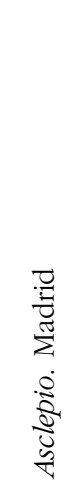 & 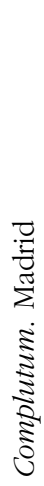 & 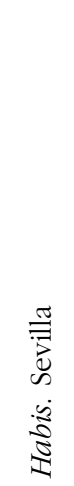 & 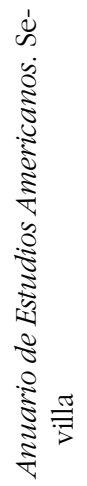 & 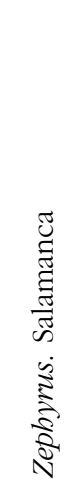 & 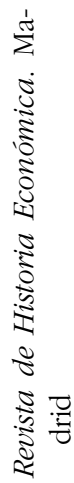 \\
\hline
\end{tabular}




\begin{tabular}{|c|c|c|c|c|c|c|c|c|c|c|c|c|c|c|c|c|}
\hline ) & $\begin{array}{l}\infty \\
\text { i } \\
\text { i }\end{array}$ & $\stackrel{0}{n}$ & $\stackrel{r}{r}$ & $\begin{array}{l}\infty \\
\infty \\
0 \\
0\end{array}$ & $\stackrel{\text { I }}{\rightarrow}$ & $\tilde{\sigma}$ & $\begin{array}{l}2 \\
\vdots \\
0\end{array}$ & $\begin{array}{l}m \\
\tilde{o}\end{array}$ & : & $\underset{f}{f}$ & oे & $\begin{array}{l}1 \\
0 \\
0\end{array}$ & in & $\stackrel{\infty}{3}$ & $\begin{array}{l}\infty \\
\text { ] } \\
0\end{array}$ & $\begin{array}{l}1 \\
0 \\
0\end{array}$ \\
\hline ్ㅡㄹ & $\begin{array}{l}\tilde{n} \\
\infty \\
0 \\
0\end{array}$ & 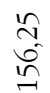 & $\begin{array}{l}\text { aे } \\
\text { ลิ }\end{array}$ & $\begin{array}{l}\stackrel{J}{J} \\
\infty \\
\infty\end{array}$ & 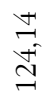 & $\frac{\tilde{n}}{\tilde{a}}$ & $\frac{2}{2}$ & $\begin{array}{l}n \\
i \\
i\end{array}$ & $\begin{array}{l}\hat{n} \\
\hat{n}\end{array}$ & $\begin{array}{l}\underset{I}{I} \\
\underset{I}{ \pm}\end{array}$ & $\begin{array}{l}\tilde{a} \\
\hat{\sigma}\end{array}$ & $\begin{array}{l}\hat{6} \\
6\end{array}$ & $\frac{7}{i n}$ & $\begin{array}{l}n \\
n \\
n\end{array}$ & $\begin{array}{l}n \\
\infty \\
\sim \\
\sim\end{array}$ & $\begin{array}{l}1 \\
0 \\
0\end{array}$ \\
\hline 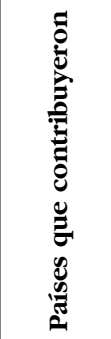 & 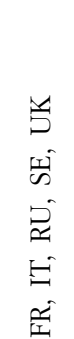 & 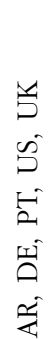 & 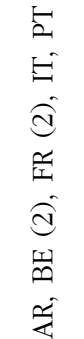 & 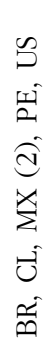 & $\begin{array}{l}0 \\
\hat{D} \\
\hat{0} \\
= \\
\hat{\oplus} \\
0 \\
0 \\
0\end{array}$ & 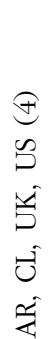 & 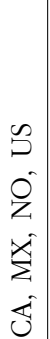 & 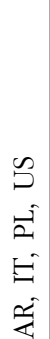 & 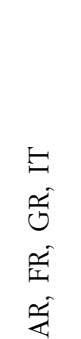 & 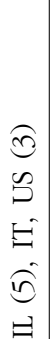 & 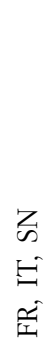 & 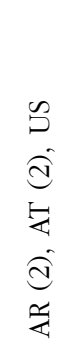 & 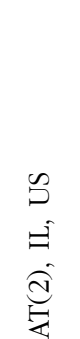 & 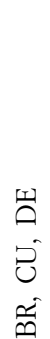 & 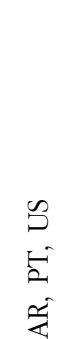 & \begin{tabular}{l}
$n$ \\
0 \\
\multirow{2}{z}{}
\end{tabular} \\
\hline 乙 & in & in & in & in & $\psi$ & 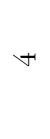 & 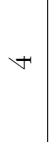 & $\checkmark$ & 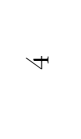 & $n$ & $n$ & $n$ & $n$ & $n$ & $n$ & $v$ \\
\hline 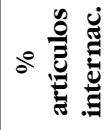 & $\begin{array}{l}\sqrt{6} \\
\underset{F}{F}\end{array}$ & $\frac{n}{n}$ & $\begin{array}{l}\hat{\imath े} \\
\text { हे }\end{array}$ & $\begin{array}{l}6 \\
6 \\
-1\end{array}$ & $\underset{\tilde{n}}{\tilde{n}}$ & $\begin{array}{l}\tilde{v} \\
\ddot{v}\end{array}$ & $\begin{array}{l}n \\
\stackrel{2}{\sigma} \\
\approx\end{array}$ & $\begin{array}{l}\infty \\
\stackrel{\infty}{\infty} \\
\stackrel{-}{\sim}\end{array}$ & $\begin{array}{l}\stackrel{\infty}{\infty} \\
\pm \\
+1\end{array}$ & $\begin{array}{l}\hat{n} \\
\approx \\
\approx\end{array}$ & $\begin{array}{l}\infty \\
0 \\
\sim \\
\sim\end{array}$ & $\begin{array}{l}\text { ה̃ } \\
\text { הิ }\end{array}$ & $\begin{array}{l}\text { bे } \\
\text { aे }\end{array}$ & $\begin{array}{l}\text { in } \\
\text { In }\end{array}$ & $\stackrel{\infty}{2}$ & $\begin{array}{l}n \\
\tilde{n} \\
n\end{array}$ \\
\hline 吕总总 & $\stackrel{n}{\sim}$ & $\stackrel{ }{\sim}$ & $r$ & 6 & $a$ & $n$ & 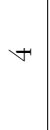 & $\checkmark$ & 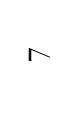 & $a$ & 0 & $\psi$ & $\psi$ & $n$ & $n$ & $\stackrel{ }{\circ}$ \\
\hline 之 & i̊ & $\approx ี$ & $\ddot{n}$ & 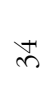 & $\widehat{\text { ̀े }}$ & iे & $\vec{\sim}$ & $\widetilde{N}$ & $\underset{\forall}{f}$ & $\curvearrowright$ & i & $\stackrel{\infty}{\sim}$ & $\vec{\sim}$ & $\stackrel{\leftrightarrow}{\sim}$ & กี & ஜি \\
\hline 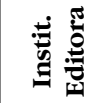 & 怘 & $\sum_{D}$ & 岕 & $\stackrel{U}{\mathscr{U}}$ & $\stackrel{U}{\mathscr{U}}$ & $\frac{U}{\tilde{U}}$ & $\stackrel{U}{\mathscr{U}}$ & $\stackrel{U}{\mathscr{U}}$ & $\sum_{S}$ & $\stackrel{\cup}{\breve{U}}$ & $\sum_{D}$ & $\sum_{\supset}$ & 芯 & 岕 & $\stackrel{U}{\widetilde{U}}$ & $\begin{array}{l}\sum \\
S\end{array}$ \\
\hline 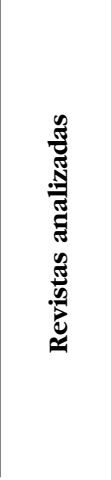 & 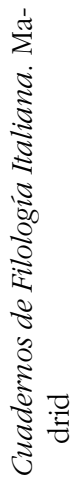 & 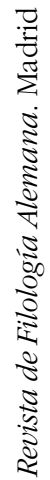 & 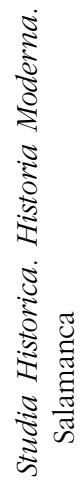 & 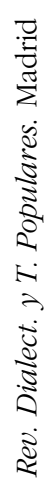 & 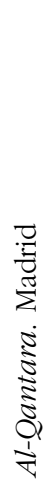 & 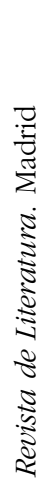 & 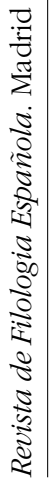 & 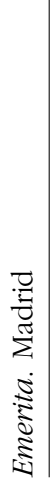 & 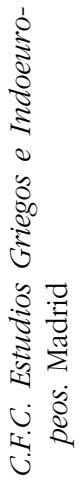 & 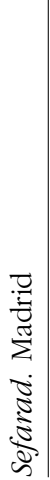 &  & 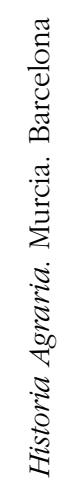 & 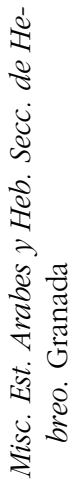 & 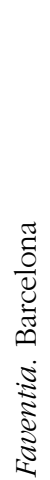 & 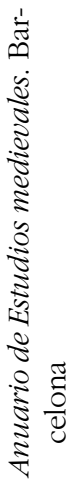 & 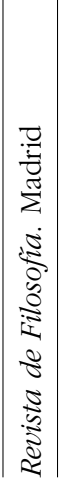 \\
\hline
\end{tabular}




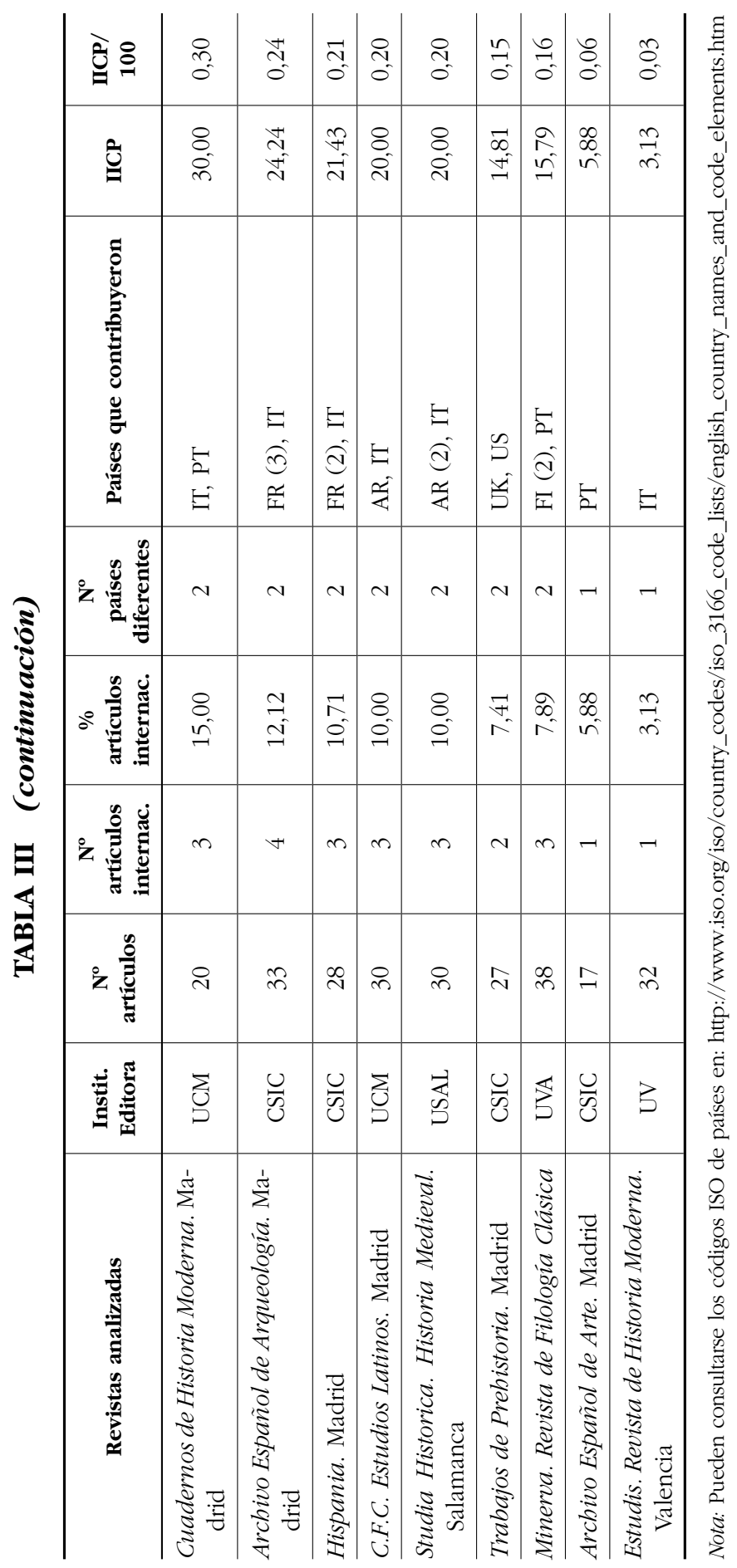




\section{FIGURA 3}

Distribución de las revistas según el porcentaje de artículos internacionales

Menos de $15 \%$ art. internac.;

Más de $50 \%$ art. internac.;

13 revistas $(35 \%)$ 6 revistas $(17 \%)$

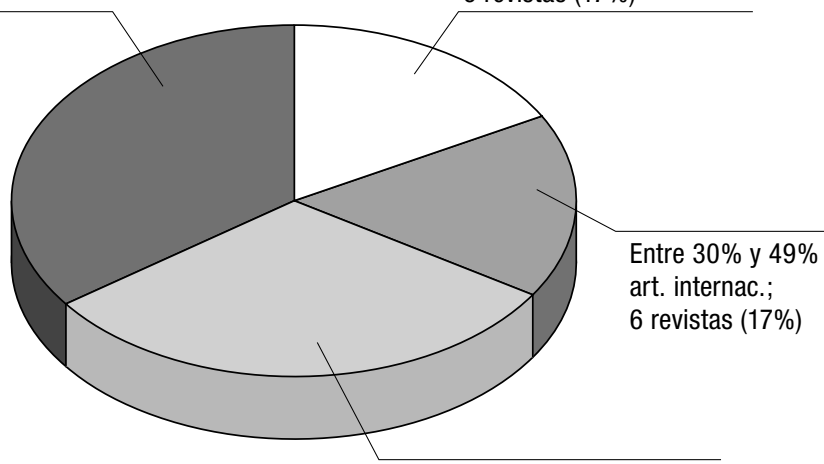

Entre $15 \%$ y $29 \%$ art. internac.;

11 revistas $(31, \%)$

\section{FIGURA 4}

Distribución de las revistas según el número de países diferentes que contribuyeron con artículos

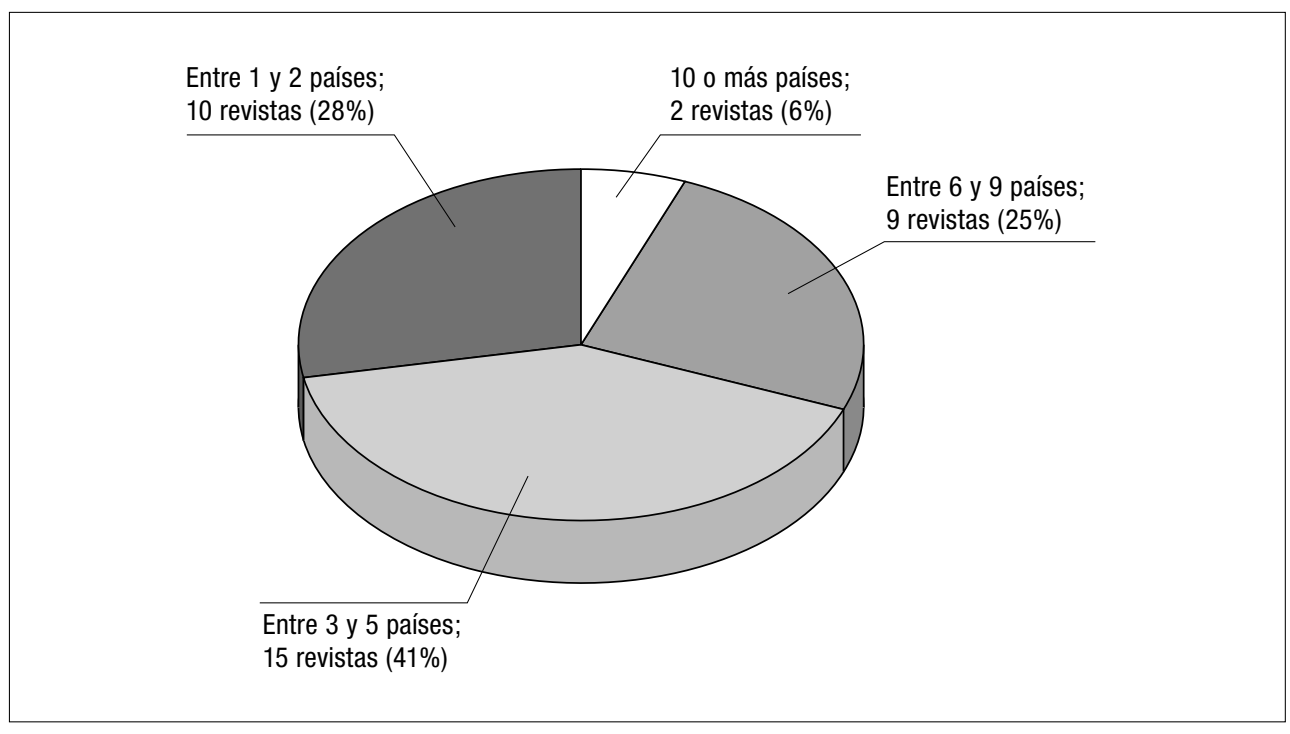


nacionales, tanto multidisciplinares como especializadas, y la posibilidad de acceder en abierto a los materiales que en ellas, se publican, son los elementos básicos para medir las probabilidades de que una revista sea, realmente, internacional en su difusión.

No todas las bases de datos dan a una revista las mismas oportunidades de ser conocida. Las bases de datos de gran prestigio hacen mucho más por la difusión de una revista que las menos prestigiosas. También es verdad que no todas las revistas tienen las mismas facilidades para ser indizadas por bases de datos especializadas, debido a que su cobertura temática no es objeto prioritario de ninguna de ellas. Sin embargo, las bases de datos multidisciplinares cubrirían este hueco para revistas que no cuentan con bases de datos de su especialidad.

Los datos de cobertura de las revistas por las diferentes bases de datos, así como su accesibilidad a texto completo en la web, se aportan en la tabla IV.

Una vez aplicados los tramos mencionados en la metodología, se obtendría la siguiente distribución:

- Revistas con visibilidad muy alta: 14 (38,89\%). Son: Archivo Español de Arte, Asclepio, Complutum, Dynamis, Emerita, Revista Española de Antropología Americana, Revista de Filosofía, Revista de Indias, Al-Qantara, Anuario de Estudios Medievales, Historia Agraria, Revista de Dialectología y Tradiciones Populares, Revista de Filología Española y Sefarad.

Además, estas 14 revistas están accesibles en abierto en Internet, bien en portales institucionales (este es el caso de las editadas por la UCM o por el CSIC), bien a través de páginas web propias o en repositorios como Dialnet o la plataforma catalana Racó. Ocho de esos títulos ofrecen un archivo de números anteriores de cinco o más años.

- Revistas con visibilidad alta: 7 revistas (19,44\%). Son: Revista de Historia Económica, Anuario de Estudios Americanos, Hispania, Trabajos de Prehistoria, Archivo Español de Arqueología, Revista Complutense de Historia de América y Zephyrus. Al igual que en el caso anterior, todas tienen edición web a texto completo, y tres de ellas cuentan con archivos que cubren períodos superiores a los cinco años.

- Revistas con visibilidad media: 10 (27,78\%). Son: Faventia, Revista de Filología Alemana, Revista de Literatura, C.F.C. Estudios Griegos e Indoeuropeos, C.F.C. Estudios Latinos, Gerión, Habis, Minerva. Revista de Filología Clásica, Estudis. Revista de Historia Moderna y Studia Historica. Historia Moderna.

La accesibilidad web en este caso es menor, pues cuatro de las diez revistas no cuentan con acceso a los textos completos, mientras que las otras seis sí (y cinco de ellas, además, permiten acceder a archivos de más de cinco años).

- Revistas con visibilidad baja 5 (13,89\%). Son: Revista de Antropología Social, Cuadernos de Historia Moderna, Studia Historica. Historia Medieval, Cuadernos de Filología Italiana y Miscelánea de Estudios Árabes y Hebraicos. 


\begin{tabular}{|c|c|c|c|c|c|c|c|c|c|c|c|c|c|}
\hline & 5 & 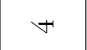 & + & $t$ & 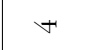 & $t$ & 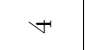 & $\checkmark$ & $n$ & 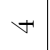 & 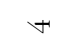 & $n$ & + \\
\hline & 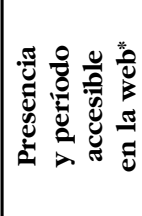 & 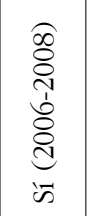 & 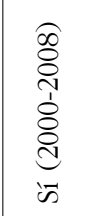 &  & 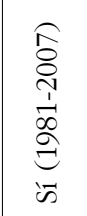 & 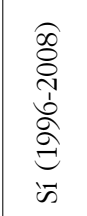 & 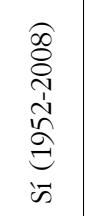 & 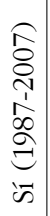 & 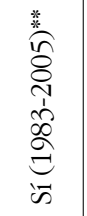 & 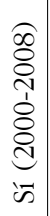 &  & 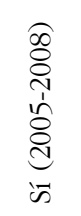 & $\begin{array}{l}\text { ô } \\
8 \\
i \\
\text { bे } \\
o \\
d \\
\text { is } \\
\text { is }\end{array}$ \\
\hline$\frac{\tilde{a}}{\tilde{3}}$ & 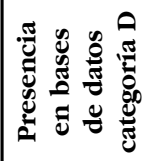 & | & | & | & $\mid$ & 1 & 1 & 1 & | & 1 & 1 & | & \\
\hline 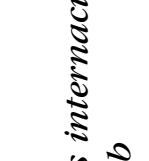 & 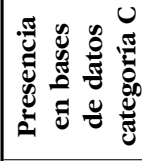 & $\begin{array}{l}\overparen{\Xi} \\
\text { 岁 } \\
\text { 至 }\end{array}$ & $\begin{array}{l}\widehat{\Xi} \\
\text { 岂 } \\
\text { 至 }\end{array}$ & $\mid$ & 1 & | & $\begin{array}{l}\overparen{\Xi} \\
\text { 岕 } \\
\text { 至 }\end{array}$ & 1 & | & 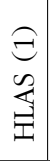 & 1 & $\begin{array}{l}\overparen{\Xi} \\
\text { 岕 } \\
\text { 至 }\end{array}$ & \\
\hline 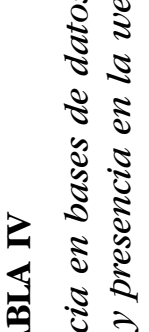 & 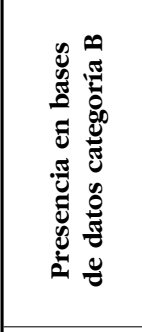 & $\begin{array}{l}\widehat{\sigma} \\
0 \\
\underline{2} \\
\dot{3} \\
\dot{3} \\
\dot{4}\end{array}$ & 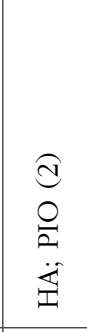 & $\begin{array}{l}\overparen{\Xi} \\
0 \\
\stackrel{0}{2}\end{array}$ & $\begin{array}{l}\overparen{\Theta} \\
0 \\
\frac{0}{2}\end{array}$ & 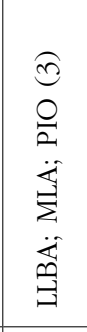 & 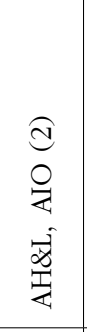 & 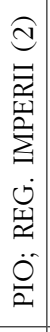 & 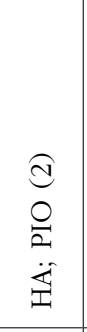 & 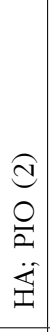 & 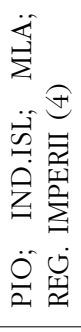 & $\begin{array}{l}\widehat{0} \\
0 \\
2 \\
\ddot{3} \\
\ddot{3} \\
0 \\
0\end{array}$ & 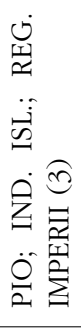 \\
\hline 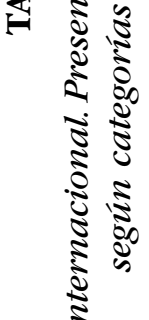 &  & 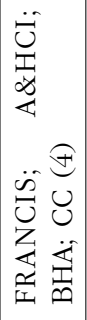 & 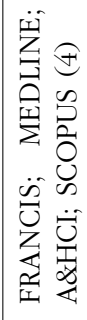 & 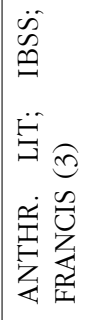 & 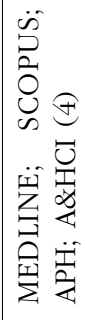 & 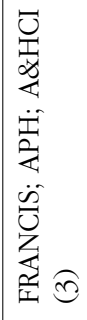 & 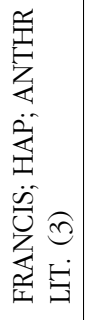 & 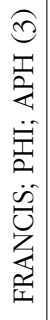 & 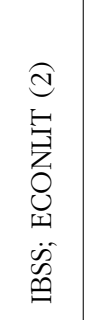 & 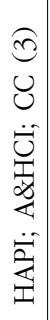 & 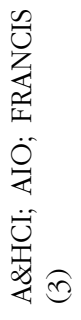 & 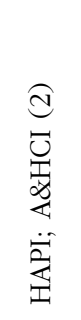 & 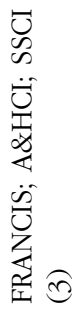 \\
\hline$\frac{\sqrt{3}}{3}$ & 总莺 & $\vec{v}$ & 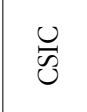 & 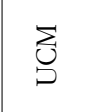 & 胥 & $\overrightarrow{\tilde{v}}$ & 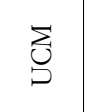 & $\begin{array}{l}\sum_{0} \\
\vdots\end{array}$ & 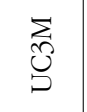 & $\frac{u}{3}$ & $\tilde{v}$ & $\overrightarrow{\widetilde{U}}$ & 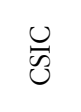 \\
\hline  & 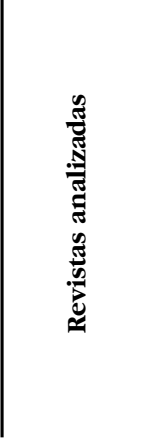 & 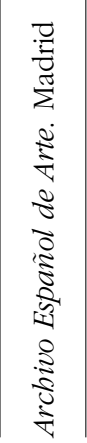 & 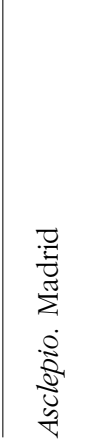 & 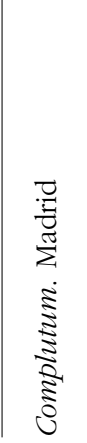 & 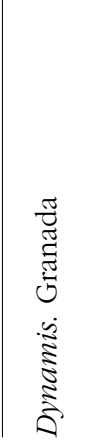 & 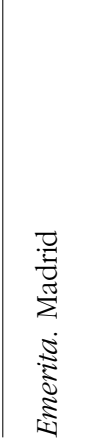 & 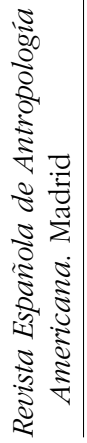 & 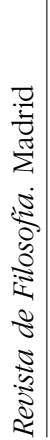 & 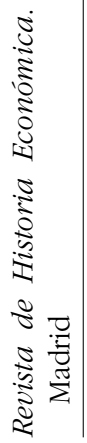 & 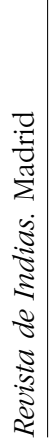 & 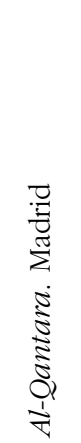 & 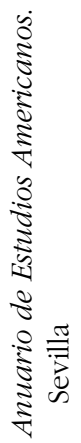 & 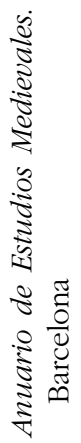 \\
\hline
\end{tabular}




\begin{tabular}{|c|c|c|c|c|c|c|c|c|c|c|c|c|c|}
\hline 5 & N & $n$ & 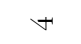 & 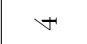 & $\checkmark$ & 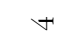 & $n$ & $n$ & $n$ & - & $v$ & $n$ & $\sim$ \\
\hline 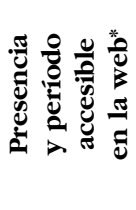 & 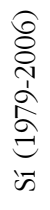 &  & 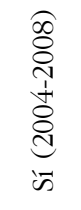 & 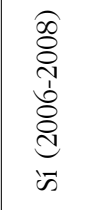 & 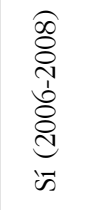 & 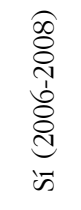 & 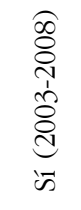 & 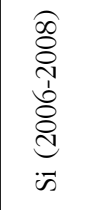 & 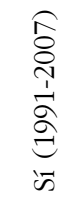 & 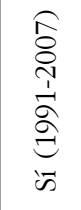 & 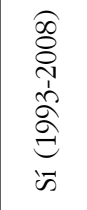 & z & 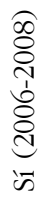 \\
\hline 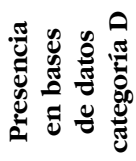 & I & $\mid$ & I & $\mid$ & & 1 & 1 & I & 1 & 1 & $\mid$ & $\mid$ & $\mid$ \\
\hline 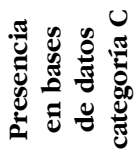 & I & 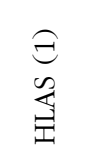 & 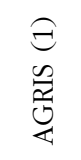 & 1 & 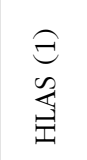 & 1 & 1 & I & 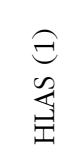 & 1 & 1 & 1 & $\mid$ \\
\hline 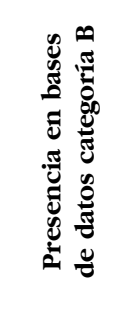 & 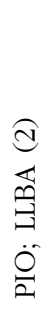 & 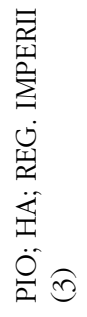 & 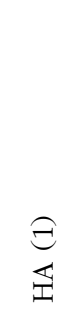 & $\begin{array}{l}\Theta \\
0 \\
0 \\
0\end{array}$ & 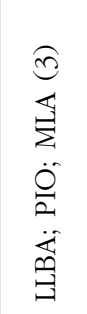 & 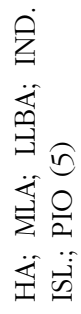 & $\begin{array}{l}\Theta \\
\stackrel{0}{2}\end{array}$ & $\begin{array}{l}\Theta \\
0 \\
0 \\
2\end{array}$ & 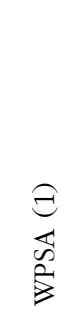 & $\begin{array}{l}\text { ङ } \\
\overleftrightarrow{4} \\
\infty \\
\ddot{4} \\
\ddot{4} \\
\ddot{4} \\
\ddot{0} \\
\ddot{4}\end{array}$ & 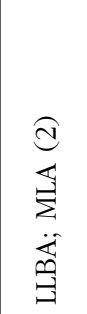 & $\begin{array}{l}\varrho \\
0 \\
\varrho \\
2\end{array}$ & $\begin{array}{l}\widehat{d} \\
0 \\
\stackrel{2}{2} \\
\dot{3} \\
\stackrel{3}{\Sigma}\end{array}$ \\
\hline 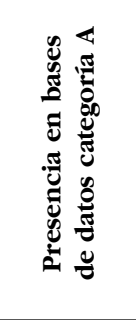 & 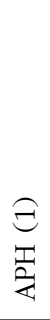 & 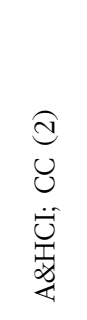 & 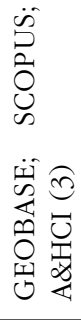 &  & 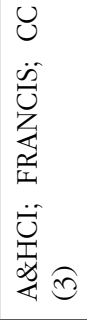 & 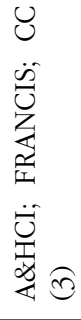 & 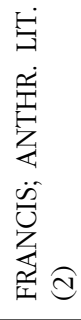 & 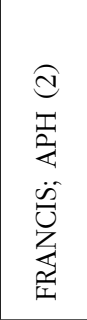 & 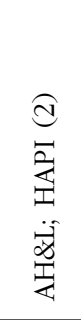 & 1 & 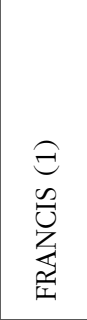 & 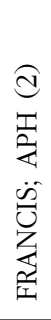 & 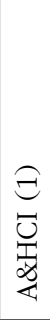 \\
\hline 苑 苛 & 空 & $\stackrel{U}{\tilde{U}}$ & 总劣 & $\stackrel{U}{\tilde{U}}$ & $\stackrel{U}{\tilde{U}}$ & $\overrightarrow{\widetilde{U}}$ & $\stackrel{U}{\widetilde{U}}$ & $\stackrel{U}{\tilde{U}}$ & $\sum_{0}$ & $\sum_{S}$ & $\sum_{S}$ & 岕 & $\vec{U}$ \\
\hline 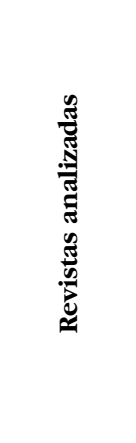 & 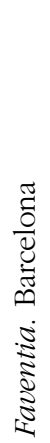 & 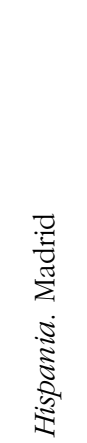 & 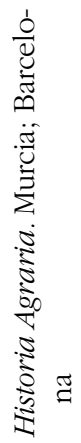 & 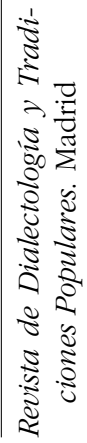 & 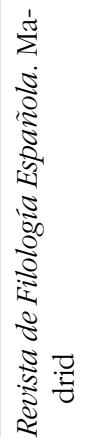 & 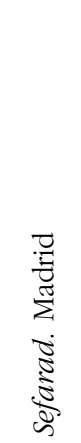 & 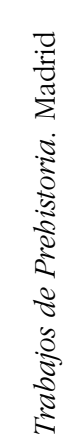 & 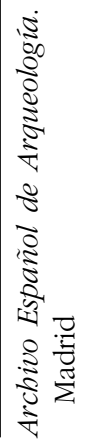 & 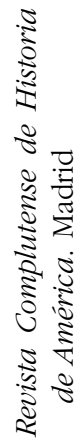 & 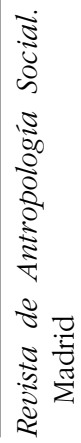 & 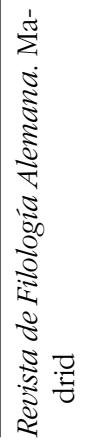 & 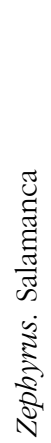 & 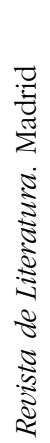 \\
\hline
\end{tabular}




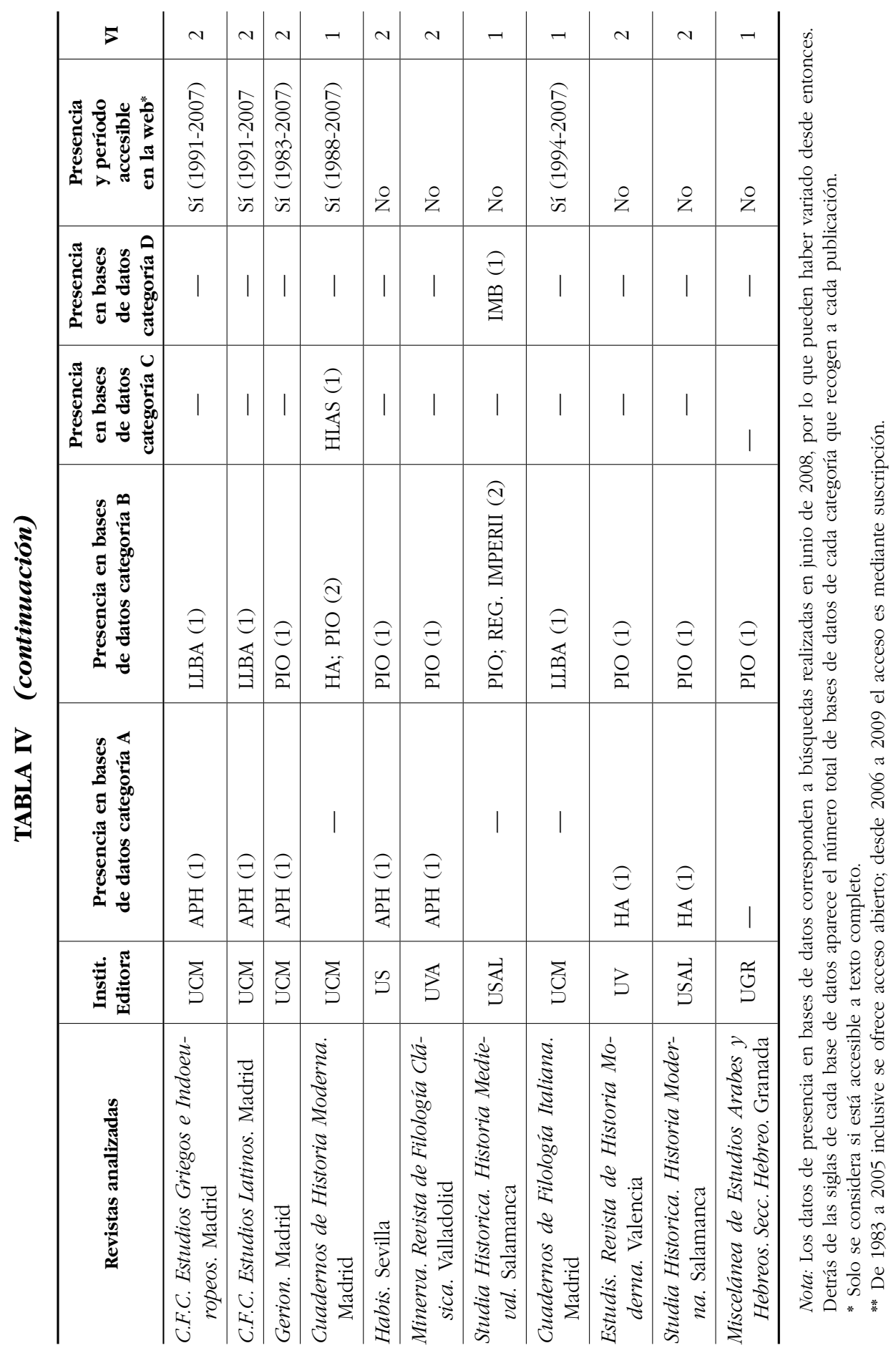


En consonancia con esa baja visibilidad, dos de las revistas no tienen acceso web a sus textos. Las otras tres sí disponen de ediciones web y con una cobertura superior a los cinco años.

La figura 5 resume las cuestiones tratadas en este apartado. Como puede verse, estamos ante un grupo de 21 revistas con buen nivel de difusión (visibilidad muy alta o alta) que, además, llevan aparejado un buen nivel de accesibilidad web, entendida aquí como posibilidad de acceder a los textos completos. El resto de revistas aún debería hacer un esfuerzo - en algunos casos, mayor que en otros- por ser más visibles tanto a través de bases de datos internacionales como a través de sus ediciones web.

\section{FIGURA 5}

Distribución de las revistas según su visibilidad en base de datos

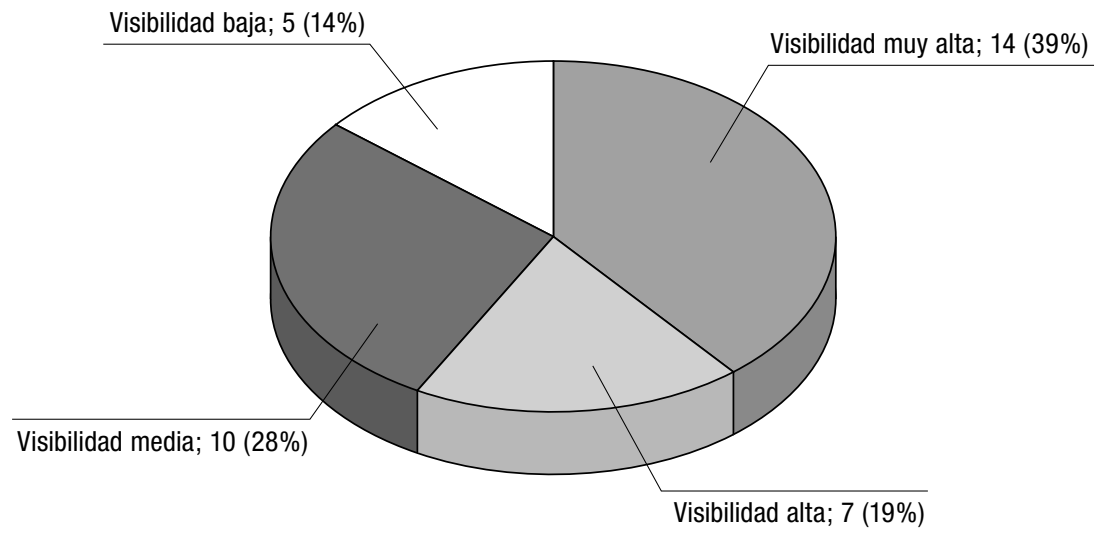

\subsection{Categorización final de la internacionalidad de las revistas a partir de las variables analizadas}

La tabla $\mathrm{V}$ permite poner en relación los valores obtenidos por cada revista para cada uno de los indicadores de internacionalidad aplicados. Empleados conjuntamente se puede obtener una idea bastante aproximada de los niveles globales de internacionalidad de las publicaciones analizadas. 


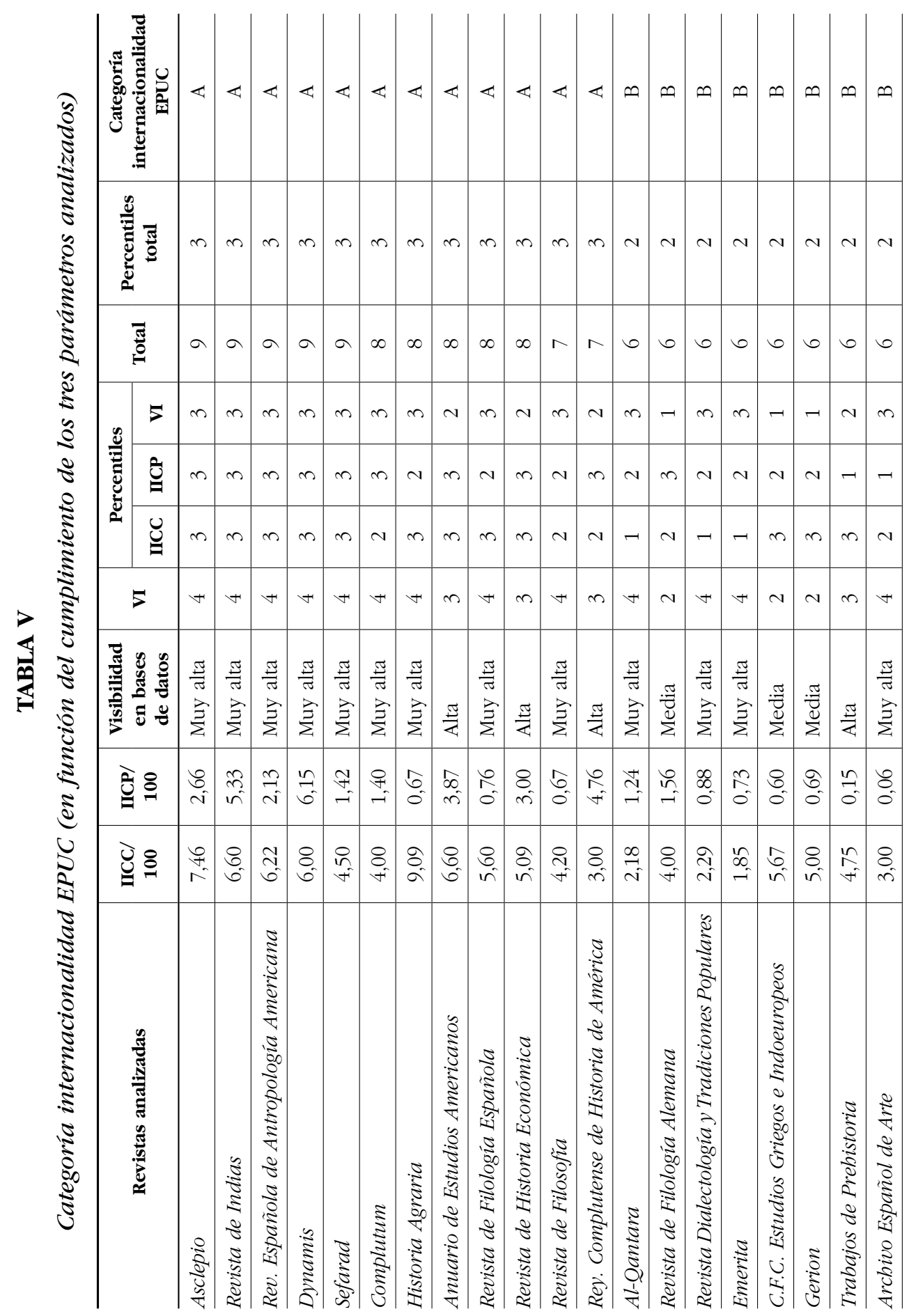




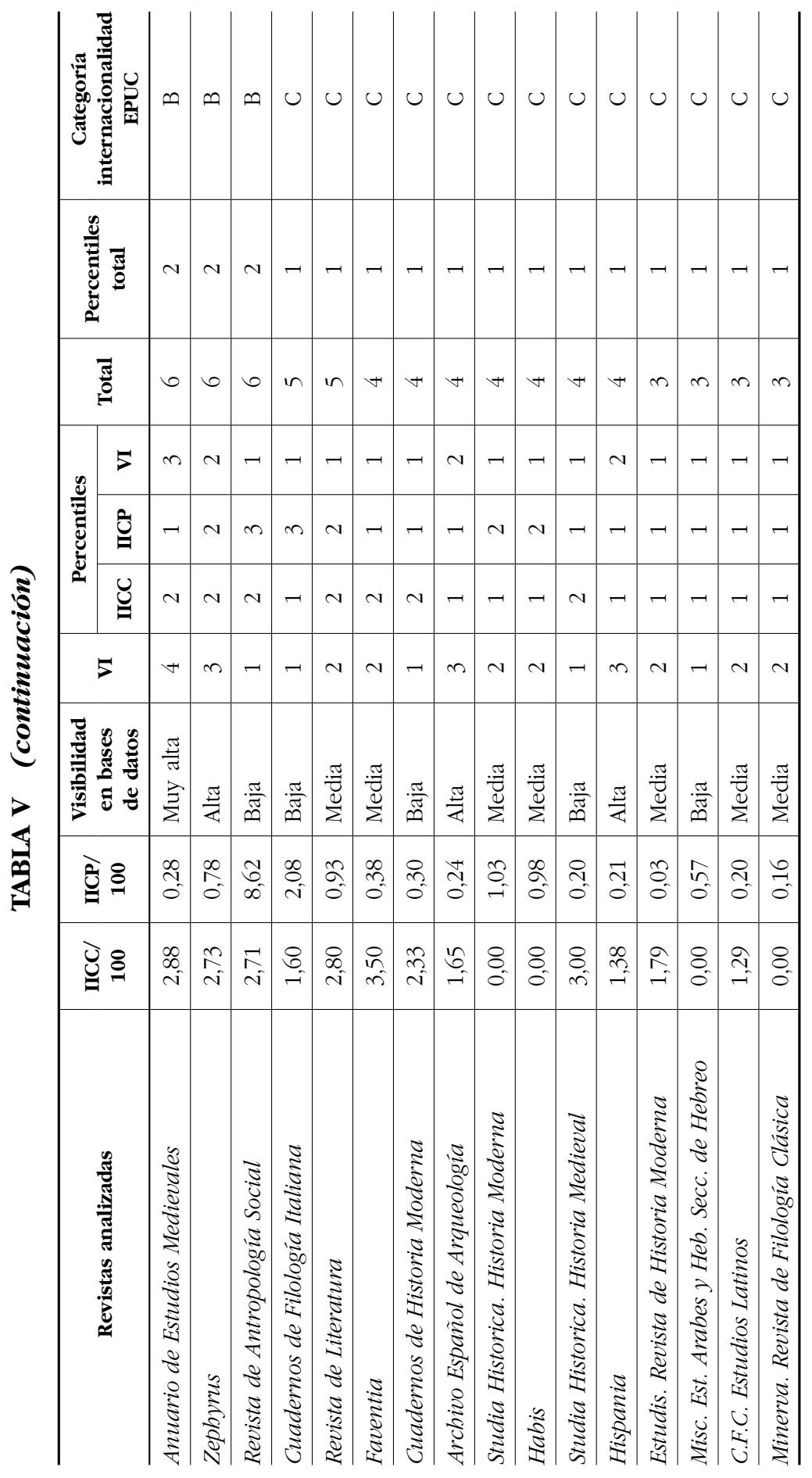


TABLA VI

Valores para el establecimiento de tramos

\begin{tabular}{l|c|c|c|c}
\cline { 2 - 5 } & IICC/100 & IICP/100 & VI & Total \\
\hline Percentil 3 & 9,09 & 8,62 & 4,00 & 9,00 \\
\hline Percentil 2 & 4,23 & 1,26 & 3,00 & 6,10 \\
\hline Percentil 1 & 2,31 & 0,59 & 2,00 & 5,00 \\
\hline
\end{tabular}

\section{Discusión y conclusiones}

\subsection{Discusión}

Si se repasa la metodología empleada en los siete trabajos reseñados en los antecedentes, se observa que hay algunas coincidencias notables: seis de ellos analizan la internacionalidad de las contribuciones publicadas; cinco tienen en cuenta la composición de los Consejos Científicos, analizando el porcentaje de miembros de instituciones extranjeras y, si bien solo dos (Pérez Álvarez-Ossorio y otros, 1977 y Malalana y otros, 2007) analizan la presencia de las revistas en las diferentes bases de datos internacionales, otros cuatro trabajos parten de la presencia de las revistas cuya internacionalidad pretenden medir, en las bases de datos WoS o en la base de datos especializada Georef (Rey-Rocha y Martín-Sempere, 2004). Es decir, la presencia en bases de datos se utiliza en seis de los siete trabajos analizados, bien para establecer la difusión internacional, bien para establecer la precondición de una internacionalidad asegurada cuyos niveles se pretenden medir, bien para asegurar una calidad incuestionable de partida.

Por otra parte, si bien la temática de las revistas como condicionante de la internacionalidad solo fue tenida en cuenta para el análisis en los trabajos de Rey-Rocha y Martín-Sempere (2004) y Malalana y otros (2007), se hizo referencia explícita a su importancia en las conclusiones de los trabajos de Ren y Rousseau (2002) y de Gutiérrez Puebla (1999).

Resumiendo, parecería bastante razonable, establecido un cierto consenso, dar por sentado que los parámetros mayoritariamente estudiados en los trabajos que se comentan son de gran importancia para la determinación de la internacionalidad de las revistas. Este relativo consenso estuvo en la base de la elección de los indicadores que se han aplicado a las revistas españolas de Humanidades que se analizan en este trabajo.

Sin embargo, al comparar los resultados obtenidos con la situación de las revistas estudiadas en las listas hechas públicas por el "Índice Europeo de Referencia para las Humanidades" (ERIH), no carece de interés examinar las discordancias en los resultados, que se reflejan en la categorización obtenida por estas revistas en las listas $\mathrm{ERIH}^{5}$. Los datos de la tabla VII ponen de relieve la dificul-

5 European Reference Index for the Humanities. Más información en: http://www.esf.org/research-areas/humanities/research-infrastructures-including-erih/erih-initial-lists.html [consultado el 10 de octubre de 2009]. 
TABLA VII

Comparación categoría EPUC vs categoría ERIH

\begin{tabular}{|c|c|c|}
\hline Revistas analizadas & $\begin{array}{c}\text { Categoría } \\
\text { internacionalidad } \\
\text { EPUC } \\
\end{array}$ & $\begin{array}{c}\text { Categoría } \\
\text { ERIH }\end{array}$ \\
\hline Asclepio & A & $\mathrm{B}$ \\
\hline Revista de Indias & A & $\mathrm{C}$ \\
\hline Rev. Española de Antropología Americana & A & $\mathrm{B}$ \\
\hline Dynamis & A & $\mathrm{C}$ \\
\hline Sefarad & A & $\mathrm{B}$ \\
\hline Complutum & A & $\mathrm{B}$ \\
\hline Historia Agraria & A & $\mathrm{B}$ \\
\hline Anuario de Estudios Americanos & A & $\mathrm{B}$ \\
\hline Revista de Filología Española & A & $\mathrm{B}$ \\
\hline Revista de Historia Económica & A & $\mathrm{B}$ \\
\hline Revista de Filosofía & A & $\mathrm{C}$ \\
\hline Rev. Complutense de Historia de América & A & $\mathrm{C}$ \\
\hline Al-Qantara & $\mathrm{B}$ & $\mathrm{B}$ \\
\hline Revista de Filología Alemana & $\mathrm{B}$ & No \\
\hline Revista Dialectología y Tradiciones Populares & $\mathrm{B}$ & $\mathrm{B}$ \\
\hline Emerita & $\mathrm{B}$ & $\mathrm{B}$ \\
\hline C.F.C. Estudios Griegos e Indoeuropeos & $\mathrm{B}$ & $\mathrm{C}$ \\
\hline Gerion & B & $\mathrm{C}$ \\
\hline Trabajos de Prebistoria & $\mathrm{B}$ & $\mathrm{B}$ \\
\hline Archivo Español de Arte & $\mathrm{B}$ & A \\
\hline Anuario de Estudios Medievales & $\mathrm{B}$ & $\mathrm{B}$ \\
\hline Zephyrus & $\mathrm{B}$ & $\mathrm{B}$ \\
\hline Revista de Antropología Social & $\mathrm{B}$ & $\mathrm{B}$ \\
\hline Cuadernos de Filología Italiana & $\mathrm{C}$ & No \\
\hline Revista de Literatura & $\mathrm{C}$ & $\mathrm{B}$ \\
\hline Faventia & $\mathrm{C}$ & $\mathrm{B}$ \\
\hline Cuadernos de Historia Moderna & $\mathrm{C}$ & $\mathrm{B}$ \\
\hline Archivo Español de Arqueología & $\mathrm{C}$ & $\mathrm{B}$ \\
\hline Studia Historica. Historia Moderna & $\mathrm{C}$ & $\mathrm{B}$ \\
\hline Habis & $\mathrm{C}$ & $\mathrm{C}$ \\
\hline Studia Historica. Historia Medieval & $\mathrm{C}$ & $\mathrm{B}$ \\
\hline Hispania & $\mathrm{C}$ & A \\
\hline Estudis. Revista de Historia Moderna & $\mathrm{C}$ & $\mathrm{B}$ \\
\hline Misc. Est. Arabes y Heb. Secc. de Hebreo & $\mathrm{C}$ & No \\
\hline C.F.C. Estudios Latinos & C & C \\
\hline Minerva. Revista de Filología Clásica & $\mathrm{C}$ & $\mathrm{C}$ \\
\hline
\end{tabular}


tad de tratamiento que, sin duda, presenta el análisis de la internacionalidad de las revistas, en este caso de Humanidades, tal y como se menciona en el primer párrafo de la introducción de este artículo.

Basta con contemplar en la tabla VII los datos relativos a cuatro de las revistas que alcanzan la máxima categoría en el ejercicio aquí realizado (Revista de Indias, Dynamis, Revista de Filosofía y Revista Complutense de Historia de América) que, sin embargo, aparecen con la categoría C en ERIH; y, al contrario, las dos únicas revistas categorizadas como A (altamente internacionales, en la terminología ERIH) (Archivo Español de Arte e Hispania) que aparecen con categorías inferiores en el sistema propuesto. Ello pone en evidencia que, según la aproximación a la internacionalidad de las revistas que se haga en cada ejercicio puede haber divergencias en los resultados y que estas divergencias pueden tener sus consecuencias en los procesos de evaluación de la actividad científica.

Estos resultados no pueden sino crear inquietud en cuantos se interesan por estos temas y obligan a profundizar en las posibles causas de esta contradictoria valoración. Se hace por tanto obligado, entrar a analizar cuál es la causa de esta situación, no sin antes tener en cuenta que los resultados que se aportan de las revistas seleccionadas para este estudio se basan, en primer lugar en los criterios previos de selección, y en segundo lugar en la aplicación de tres parámetros relevantes en orden a la valoración de la internacionalidad: la composición internacional de los consejos, el porcentaje de contribuciones internacionales y la visibilidad internacional medida en la presencia en bases de datos internacionales de prestigio. Los resultados han sido buenos cuando la respuesta a los tres parámetros analizados también lo era. Pero hay que pensar que pueden aplicarse además otro tipo de parámetros que pueden hacer variar los resultados y también que la categorización final variaría si se introdujeran muchos más títulos en la muestra. Además, el ejercicio realizado tiene limitaciones en cuanto al volumen de números analizados de cada publicación: únicamente los tres últimos números editados de cada revista. Obviamente, si se analizaran más números podrían detectarse variaciones en los resultados. Todas estas limitaciones, si bien relativizan los resultados obtenidos, no anulan su fuerza expresiva para poner de manifiesto la dificultad de la valoración de la internacionalidad, objetivo de este trabajo.

La dificultad en la comparación de las categorías se deriva del desconocimiento de los detalles metodológicos con que ERIH obtiene sus listados, si bien se ha trabajado con el material que es público: enunciados teóricos de la estimación de la visibilidad de las revistas. Esto hace más complicado que puedan detectarse los factores en los que se basan las discrepancias de los resultados, en los casos que resultan llamativos de la tabla VII.

De modo que si, en aras a una mayor clarificación, se intentan analizar las razones que hacen que, por ejemplo, las cuatro revistas mencionadas anteriormente (Dynamis, Revista de Indias, Revista de Filosofía o Revista Complutense de Historia de América) que alcanzan la categoría más alta relativa a internacionalidad según el modelo propuesto, son consideradas como "nacionales" en las 
listas ERIH, como refleja la categoría C que se les asigna, y si además pueden aducirse los méritos concretos comprobables que avalan su posición en la categorización EPUC, habrá que intentar buscar las razones de esta diferencia de resultados recurriendo a parámetros o criterios de valoración que, estando incluidos en el proyecto ERIH, no se han tenido en cuenta en este trabajo. De otra manera, con los datos públicos disponibles, es difícil encontrar las razones de su categorización como C en las listas ERIH.

Dynamis, por referirnos a uno de los casos que plantean diferencias en las dos categorizaciones, es una revista editada por la Universidad de Granada desde el año 1981 y que está recogida por las siguientes bases de datos internacionales: MEDLINE; SCOPUS; A\&HCI; APH y PIO. Es una revista que selecciona sus originales para publicación mediante un sistema de revisores expertos, externos a la Universidad de Granada y al propio Consejo de Redacción. Su Consejo Científico tiene un 66,67\% de miembros internacionales y participan en él 10 países diferentes, incluyendo a España.

Publicó en los tres números analizados un total de 39 trabajos, 20 de los cuales estaban firmados por autores de instituciones de 12 países diferentes, 7 de ellos latinoamericanos (Argentina, Brasil, Colombia, Costa Rica, Cuba, México y Perú) y otros 5 europeos y norteamericanos (Alemania, Canadá, Reino Unido, Irlanda y los Estados Unidos).

Podría decirse que la presencia activa de colaboraciones de tantos países diferentes, así como la presencia en las bases de datos internacionales más prestigiosas podrían avalar con creces su calidad académica. Ahora bien, el "amplio consenso en el área sobre su estatus y su visibilidad internacional" que pide ERIH (véase información relativa a ERIH en el apartado de antecedentes) ¿cómo se mide? ¿cómo se manifiesta en la práctica, de manera que un comité de cuatro o cinco personas puedan avalarlo? Es indudable, en este caso, que la capacidad demostrada por Dynamis para atraer autores de tan diferentes instituciones y países es un buen aval del "consenso en el área" sobre su internacionalidad. Por otra parte, se exige apertura a las contribuciones no solicitadas, y tanto en el caso de Dynamis como en el de las demás revistas analizadas aquí, la mayoría de las contribuciones no son solicitadas. Esto suele ser, al menos en las revistas científicas españolas, una excepción, afectando de manera especial a los números monográficos de algunas revistas cuya coordinación se deja a un editor/coordinador que suele solicitar trabajos a especialistas del tema objeto del monográfico. Por otra parte, este hecho no obvia el sometimiento de los trabajos al informe preceptivo de al menos dos revisores externos. En cuanto a la puntualidad en la edición de acuerdo con una periodicidad previamente declarada y con la que el editor se compromete, la revista Dynamis es anual y cumple sus plazos escrupulosamente. Quedaría la última exigencia: ¿recibe la revista suficientes originales como para poder elegir de entre ellos los de incuestionable calidad? De momento, al menos en España y en el ámbito de las Humanidades y las Ciencias Sociales, son aún contadas las revistas que aportan datos sobre los originales recibidos y las tasas de aprobación o rechazo. De manera que los datos hay que obtener- 
los normalmente dirigiéndose a los editores que, sin hacerlos públicos, pueden ser más o menos fiables. Ahora bien, los indicadores analizados, pueden dar fe de que los contenidos tienen una calidad científica asegurada, lo que induce a pensar que el sistema de referees utilizado funciona.

Quizás pueda pensarse que los tres indicadores aplicados a las revistas, previamente seleccionadas por su calidad y su cumplimiento de las normas internacionales, no sean suficientes para calificar de internacional a una revista, pero las condiciones adicionales que plantea ERIH no están definidas de manera inequívoca, no se explica la metodología utilizada para evaluarlas, y, en definitiva, la garantía de igualdad de oportunidades para todas las revistas es bastante aleatoria, dado el margen de discrecionalidad.

Lo mismo cabría decir en relación con las otras tres revistas en las que se observa la misma situación (poco internacionales para ERIH a diferencia de los resultados de la propuesta de este trabajo). Sin embargo, cuando una revista tan prestigiosa como Hispania, sufre el efecto contrario (ha sido categorizada como A por ERIH, pero no resulta altamente internacional según la categorización EPUC), puede buscarse la explicación en los tres números analizados; tiene un porcentaje de trabajos firmados por autores de instituciones extranjeras muy bajo - solo un 10,1\% - y las instituciones firmantes pertenecen solo a dos países diferentes. Quizás este resultado habría variado si el análisis se hubiera hecho con más números y esta crítica puede ser aceptable en nuestro trabajo.

Por otra parte, si se repasan los contenidos de Hispania y de Revista de Indias (como ejemplos claros de divergencia en las categorizaciones), en un intento por observar una variable que resulta determinante en la internacionalidad, la explicación a estas diferencias aparece bastante nítida: los contenidos de la $R e$ vista de Indias son, por su propia naturaleza, internacionales, pues su tema nuclear es América y, más en concreto, Iberoamérica. No sucede lo mismo con Hispania, donde los trabajos que se publican sobre Historia de España son mayoritarios, aunque sin limitarse a ningún ámbito geográfico, como los editores afirman: "Hispania. Revista Española de Historia es una publicación cuatrimestral dedicada al estudio de las sociedades en las épocas medieval, moderna y contemporánea. Sus páginas están abiertas a investigaciones originales comprendidas en estos tres amplios estratos cronológicos, sin limitaciones en cuanto a temática específica ni a su ámbito geográfico" ${ }^{6}$.

La diferencia de temas de estudio de una y otra, mas allá de las declaraciones, se refleja lógicamente en el tipo y el origen de las contribuciones que reciben, y explica especialmente los datos presentados en los cuadros. Esto no disminuye en absoluto la calidad de Hispania. En cambio, no hay explicación razonable para la categorización en ERIH de revistas como Dynamis o la Revista de Indias como C. ¿Cuáles son las razones para ello? Si la reproductibilidad de los trabajos científicos es una exigencia del buen hacer, sería deseable y de esperar, que las

${ }^{6}$ Véase el registro correspondiente en Latindex: http://www.latindex.org/larga.php?opcion= $1 \&$ folio $=5970$ [consultado el 10 de octubre de 2009]. 
categorizaciones acordadas fueran autoexplicables, con los criterios de evaluación aplicados en la mano.

Está el criterio superior de los "pares" o, dicho de otro modo, de la opinión de los especialistas. En las Ciencias Sociales y en las Humanidades es difícil que la valoración de los expertos aporte resultados totalmente objetivos, en un escenario cruzado de escuelas de pensamiento, tradiciones culturales, equilibrios políticos entre países y lenguas, mayor o menor lejanía de las culturas latinas, anglosajonas, eslavas, no siempre bien representadas en los comités evaluadores, etc. La consulta a los expertos es importantísima, pues solo desde el conocimiento de las materias específicas puede enjuiciarse la calidad de contenidos de una manera directa. Pero asegurar que la valoración hecha por ellos está exenta de sesgos, solo es posible cuando los consultados son una muestra tan amplia y tan representativa de cada especialidad, que pueda garantizarse que dichos sesgos están debidamente neutralizados.

Por todo lo anterior, sería muy recomendable un esfuerzo de clarificación, por parte del proyecto ERIH, tanto en la descripción de los criterios exigidos como en el método de estimación de su cumplimiento, de manera que quede claro para todos que el proceso de valoración tiene unas normas públicas y bien definidas. Así, todos los concernidos, estén o no de acuerdo con ellas, podrán esgrimir los datos probatorios del cumplimiento de los diferentes parámetros, cuando éstos no hubieran sido bien estimados. Es decir, sería necesario que cualquier proceso de evaluación, como se requiere en todo trabajo científico, tenga la condición de la reproducibilidad, de forma que cualquiera pueda repetir los procesos por los que una revista ha sido categorizada de una determinada manera.

\subsection{Conclusiones}

Para finalizar, y a modo de resumen, tres serían las conclusiones más claras de este trabajo:

a) Los resultados del análisis de las 36 revistas españolas de Humanidades estudiadas muestran que un 33,3\% de los títulos presentan rasgos indiscutibles de internacionalidad bien asentada: por la estructura de sus consejos, por las aportaciones de variados países en un alto porcentaje de las contribuciones publicadas y por una amplia visibilidad internacional facilitada por su presencia en prestigiosas bases de datos tanto multidisciplinares como especializadas. Otro 30,5\% de las revistas tienen características de internacionalidad claras, aunque no tan contundentes. Finalmente, el 36\% restante serían revistas de indiscutible calidad, pero de escasa proyección internacional.

b) La complejidad inherente a la valoración y la medición de los niveles de internacionalidad de las revistas de Humanidades aconsejaría en lo posi-

Rev. Esp. Doc. Cient., 33, 3, julio-septiembre, 341-377, 2010. ISSN: 0210-0614. doi:10.3989/redc.2010.3.735 
ble ampliar los parámetros a analizar con objeto de cubrir los diferentes aspectos que pueden incidir de manera clara en la internacionalidad de una revista: además de los más frecuentemente utilizados en la literatura especializada, cabría analizar la internacionalidad de los temas tratados en los artículos, la internacionalidad de las referencias aportadas en los aparatos críticos y las citas recibidas a los trabajos publicados, cuando esto fuera posible. Además, un análisis de presencias de "ida y vuelta" tal y como analiza Uzún, sería un complemento importante para los estudios de internacionalidad referidos a disciplinas o subdisciplinas concretas.

c) Por último, y a la vista de las discrepancias de resultados con otras experiencias de valoración de la internacionalidad de las revistas de Humanidades, se concluye en la necesidad de que los sistemas, productos o listas que se puedan elaborar para discernir los niveles de internacionalidad de las publicaciones, definan con claridad los indicadores que aplican así como la metodología de aplicación de los mismos, de manera que los resultados puedan ser reproducibles. Dada la incidencia que estos productos tienen en los sistemas de evaluación científica, es importante facilitar al máximo la comparabilidad de los resultados y la máxima objetividad y claridad en las metodologías aplicadas.

Si se tuvieran en cuenta las recientes incorporaciones de algunas de las revistas analizadas especialmente a la base de datos Scopus en Marzo de 2009 (véase Anexo II), algunos de los resultados de la categorización podrían haberse visto afectados, lo que no menoscabaría ninguna de las conclusiones fundamentales de este trabajo en relación con la dificultad de determinar los niveles de internacionalidad de las revistas y de la imperiosa necesidad de mejorar la comunicación sobre la metodología seguida en la aplicación de los indicadores utilizados para la elaboración de las diferentes listas, ranking, etc., en aras de una mayor claridad y de garantizar la reproducibilidad de las evaluaciones efectuadas.

\section{Bibliografía}

Alcain-Partearroyo, M. D.; Román-Román, A., y Giménez-Toledo, E. (2008). Categorización de las revistas españolas de Ciencias Sociales y Humanidades en RESH. Revista Española de Documentación Científica, vol. 31, (1), 85-95.

Buela Casal, G.; Perakakis, P.; Taylor, M., y Checa, P. (2006). Measuring internationality: reflections and perspectives on academic journals. Scientometrics, vol. 67 (1), 45-65.

Delgado López-Cozar, E.; Ruiz-Pérez, R., y Jiménez-Contreras, E. (2006). La edición de revistas científicas. Directrices, criterios y modelos de evaluación. Madrid: FECYT. http://recyt.fecyt.es/documentos/Fecyt.pdf [consultado el 15 de septiembre de 2009].

ERIH Summary guidelines (s.d.). http://www.esf.org/research-areas/humanities/researchinfrastructures-including-erih/erih-initial-lists.html [consultado el 1 de septiembre de 2009]. 
Gutiérrez Puebla, J. (1999). Las revistas internacionales de Geografía: internacionalización e impacto. Boletín de la Sociedad de Geógrafos Españoles (27), 117-134.

Malalana Ureña, A.; Román-Román, A., y Rubio-Liniers, M. C. (2007). Visibilidad internacional de las revistas españolas de Historia. Scripta Nova. [En línea]. Barcelona: Universidad de Barcelona, vol. 11 (234). http://www.ub.es/geocrit/sn/sn-234.htm [consultado el 28 de mayo de 2009].

Pérez Álvarez-Ossorio, J. R.; Gómez, I., y Martín-Sempere, M. J. (1997). International visibility of domestic scientific literature. Journal of Information Science, vol. 23 (1), 98-101.

Ren, S., y Rousseau, R. (2002). International visibility of Chinese scientific journals. Scientometrics, vol. 53 (3), 389-405.

Rey-Rocha, J., y Martín-Sempere, M. J. (2004). Patterns of the foreign contributions in some domestic vs. international journals on Earth Sciences. Scientometrics, vol. 59 (1), 95115 .

Román, A. (coord.) (2001). La edición de revistas científicas: guía de buenos usos. Madrid: CINDOC.

Uzún, A. (2004). Assessing internationality of scholarly journals through foreign authorship patterns: the case of major journals in Information Science and Scientometrics. Scientometrics, vol. 61 (3), 457-465.

\section{Anexo I. Bases de datos consultadas. Acrónimos y desarrollo}

\begin{tabular}{l|l}
\hline \multicolumn{1}{c|}{ Acrónimo } & \multicolumn{1}{c}{ Nombre desarrollado } \\
\hline A\&HCI & Arts \& Humanities Citation Index \\
\hline AA & Art Abstracts \\
\hline AGRIS & International Information System for the Agricultural Sciences and Technology \\
\hline AH\&L & America History \& Life \\
\hline AIO & Anthropological Index Online \\
\hline ANTHR. LIT. & Anthropological Literature \\
\hline APH & L'Année Philologique \\
\hline BHA & The Bibliography of the History of Art \\
\hline CC & Current Contents \\
\hline ECONLIT & The American Economic Association's electronic bibliography \\
\hline FRANCIS & (A unique bibliographic database in Humanities and Social Sciences) \\
\hline GEOBASE & The Bibliographic database for the Earth, Geographical and Ecological Sciences \\
\hline HA & Historical Abstracts \\
\hline HAPI & Hispanic American Periodical Index \\
\hline HLAS & Handbook of Latin American Studies \\
\hline IBSS & International Bibliography of the Social Sciences \\
\hline
\end{tabular}




\begin{tabular}{l|l}
\hline \multicolumn{1}{c|}{ Acrónimo } & \multicolumn{1}{c}{ Nombre desarrollado } \\
\hline IBZ & $\begin{array}{l}\text { Internationale Bibliographie der Geistes-Und Sozialwissenschaftlichen Zeitschif- } \\
\text { tenliteratur }\end{array}$ \\
\hline IMB & International Medieval Bibliography \\
\hline IND. ISL. & Index Islamicus \\
\hline LLBA & Linguistics and Language Behaviour Abstracts \\
\hline MEDLINE & (Base de datos de la National Library of Medicine) \\
\hline MLA & Modern Language Association Bibliography \\
\hline PHI & The Philosopher's Index \\
\hline PIO & Periodical Index Online \\
\hline RBPH & Répertoire Bibliographique de la Philosophie de Louvain \\
\hline REG. IMPERII & Regesta Imperii \\
\hline SA & Sociological Abstracts \\
\hline SCI & Science Citation Index \\
\hline SCOPUS & (Scopus is the largest abstract and citation database of peer-reviewed literature) \\
\hline SSA & Social Services Abstracts \\
\hline SSCI & Social Sciences Citation Index \\
\hline WPSA & Worldwide Political Science Abstracts \\
\hline
\end{tabular}

\section{Anexo II. Datos actualizados de la cobertura de revistas por parte de las bases de datos}

Datos actualizados con la Scopus Master List de 20 de marzo de 2009 y relación de revistas españolas en la WoS de enero 2009. La incorporación de revistas a IBZ (K. G. Saur Verlag) fue comunicada a EPUC en abril de 2009. Se muestran en negrita las bases de datos que incluyeron a las revistas con posterioridad a la búsqueda realizada para la elaboración del artículo.

\begin{tabular}{l|l}
\hline \multicolumn{1}{c|}{ Revistas seleccionadas } & \multicolumn{1}{c}{ Bases de datos } \\
\hline Al-Qantara. Madrid & $\begin{array}{l}\text { PIO; A\&HCI; IND.ISL.; MLA; FRANCIS; AIO; } \\
\text { REG. IMPERII; RBPH; IBZ; SCOPUS }\end{array}$ \\
\hline Anuario de Estudios Americanos. Sevilla & HAPI; HLAS; AIO; HA; PIO; A\&HCI \\
\hline Anuario de Estudios Medievales. Barcelona & $\begin{array}{l}\text { FRANCIS; PIO; IND. ISL.; REG. IMPERI; A\&HCI; } \\
\text { SSCI }\end{array}$ \\
\hline Archivo Español de Arqueología. Madrid & FRANCIS; PIO; APH; A\&HCI; IBZ \\
\hline Archivo Español de Arte. Madrid & $\begin{array}{l}\text { FRANCIS; AA; A\&HCI; BHA; HA; HLAS; CC; } \\
\text { PIO; IBZ; SCOPUS }\end{array}$ \\
\hline Asclepio. Madrid & $\begin{array}{l}\text { HA; FRANCIS; HLAS; MEDLINE; PIO; A\&HCI; } \\
\text { SCOPUS }\end{array}$ \\
\hline
\end{tabular}




\begin{tabular}{|c|c|}
\hline Revistas seleccionadas & Bases de datos \\
\hline C.F.C. Estudios Griegos e Indoeuropeos. Madrid & LLBA; APH \\
\hline C.F.C. Estudios Latinos. Madrid & LLBA; APH \\
\hline Complutum. Madrid & FRANCIS; IBSS; ANTHR. LIT.; PIO \\
\hline Cuadernos de Filología Italiana. Madrid & LLBA \\
\hline Cuadernos de Historia Moderna. Madrid & HA; HLAS; PIO \\
\hline Dynamis. Granada & $\begin{array}{l}\text { FRANCIS; HA; MEDLINE; PIO; SCOPUS; APH; } \\
\text { A\&HCI; SSCI; SCI }\end{array}$ \\
\hline Emerita. Madrid & $\begin{array}{l}\text { FRANCIS; LLBA; MLA; PIO; APH; A\&HCI; IBZ; } \\
\text { SCOPUS }\end{array}$ \\
\hline Estudis. Revista de Historia Moderna. Valencia & $\mathrm{HA} ; \mathrm{PIO}$ \\
\hline Faventia. Barcelona & PIO; LLBA; APH \\
\hline Gerion. Madrid & APH; PIO \\
\hline Habis. Sevilla & PIO; APH; IBZ \\
\hline Hispania. Madrid & $\begin{array}{l}\text { A\&HCI; HLAS; PIO; CC; HA; REG. IMPERII; IBZ; } \\
\text { SCOPUS }\end{array}$ \\
\hline Historia Agraria. Murcia; Barcelona & HA; AGRIS; GEOBASE; SCOPUS; A\&HCI; IBZ \\
\hline Minerva. Revista de Filología Clásica. Valladolid & $\mathrm{PIO} ; \mathrm{APH}$ \\
\hline Misc. Est. Ar. y Heb. Secc. Hebreo. Granada & PIO; IBZ \\
\hline Rev Dialectología y T. Populares. Madrid & AIO; A\&HCI; FRANCIS; CC; PIO; IBZ; SCOPUS \\
\hline Rev. Complutense de Historia de América. Madrid & HAPI; HLAS; HA; WPSA; AH\&L; PIO; SCOPUS \\
\hline Rev. Española de Antropología Americana. Madrid & HA; AH\&L; HAPI; HLAS; AIO; PIO; ANTHR. LIT. \\
\hline Revista de Antropología Social. Madrid & $\mathrm{HA} ; \mathrm{AIO} ; \mathrm{SA} ; \mathrm{SSA}$ \\
\hline Revista de Filología Alemana. Madrid & LLBA; MLA; IBZ; SCOPUS \\
\hline Revista de Filología Española. Madrid & $\begin{array}{l}\text { A\&HCI; FRANCIS; HLAS; LLBA; PIO; CC; MLA; } \\
\text { IBZ; SCOPUS }\end{array}$ \\
\hline Revista de Filosofia. Madrid & FRANCIS; PHI; PIO; APH; REG. IMPERII; RBPH \\
\hline Revista de Historia Económica. Madrid & HA; IBSS; PIO; ECONLIT; SSCI; IBZ \\
\hline Revista de Indias. Madrid & HAPI; HLAS; HA; A\&HCI; PIO; CC; IBZ; SCOPUS \\
\hline Revista de Literatura. Madrid & MLA; A\&HCI; PIO; IBZ; SCOPUS \\
\hline Sefarad. Madrid & $\begin{array}{l}\text { A\&HCI; FRANCIS; HA; MLA; LLBA; IND. ISL.; } \\
\text { CC; PIO; RBPH; IBZ; SCOPUS }\end{array}$ \\
\hline Studia Historica. Historia Medieval. Salamanca & IMB; PIO; REG. IMPERII \\
\hline Studia Historica. Historia Moderna. Salamanca & $\mathrm{HA} ; \mathrm{PIO}$ \\
\hline Trabajos de Prebistoria. Madrid & $\begin{array}{l}\text { FRANCIS; ANTHR. LIT.; PIO; SSCI; A\&HCI; } \\
\text { SCOPUS }\end{array}$ \\
\hline Zephyrus. Salamanca & FRANCIS; PIO; APH; IBZ \\
\hline
\end{tabular}

